\title{
Limit of Thermal-Vibration Noise in Magnetic Field Detection with Magnetoelectric-Composite Cantilevers
}

\author{
Matthias C. Krantz ${ }^{*}$ and Martina Gerken \\ Institute of Electrical Engineering and Information Technology, Kiel University, 24143 Kiel, Germany
}

(Received 12 June 2019; revised manuscript received 9 January 2020; accepted 3 April 2020; published 20 May 2020)

\begin{abstract}
The fundamental thermal limit of magnetic field sensing using the resonance-enhanced magnetoelectric (ME) effect in strain-coupled ferroic composite cantilevers is investigated theoretically with results applied to $\left(\mathrm{Fe}_{90} \mathrm{Co}_{10}\right)_{78} \mathrm{Si}_{12} \mathrm{~B}_{10} / \mathrm{Si} / \mathrm{AlN}$ using experimental material parameters. An analytic theory of the ME response and noise from thermal vibrations, Johnson-Nyquist noise from dielectric losses in AlN, and amplifier electronics is given to yield the magnetic field detection limit for the first bending mode. A systematic investigation of scaling behavior of ME response, sensitivity, noise components, and detection limit with cantilever size, layer thicknesses, quality factor $Q_{f}$, and resonance frequency $f$ is presented emphasizing the low-frequency small-size regime essential for biomagnetic sensing applications. Thermal vibrations are found to dominate all investigated noise sources for practical composite sizes except at low quality factors. Detection limit scaling inversely with functional layer thickness, as $1 /$ size $^{2}$ for constant $f$ and $Q_{f}$, and as $Q_{f}^{-1 / 2}$ (constant size and $f$ ) is found. Achievable detection limits below $100 \mathrm{fT} / \mathrm{Hz}^{1 / 2}$ are predicted for $\left(\mathrm{Fe}_{90} \mathrm{Co}_{10}\right)_{78} \mathrm{Si}_{12} \mathrm{~B}_{10} / \mathrm{Si} / \mathrm{AlN}$ composite cantilevers at sizes below $10 \mathrm{~mm}$ (and even lower without size constraint) with $f=1 \mathrm{kHz}, Q_{f}=1000$, and $\left(\mathrm{Fe}_{90} \mathrm{Co}_{10}\right)_{78} \mathrm{Si}_{12} \mathrm{~B}_{10}$ layers of $50 \mu \mathrm{m}$. The scaling behavior implies the absence of an absolute detection limit for thick ferroic layers within the range of validity of the experimental material parameters observed at $\sim 2 \ldots 4 \mu \mathrm{m}$.
\end{abstract}

DOI: 10.1103/PhysRevApplied.13.054047

\section{INTRODUCTION}

Significant research interest has recently emerged in magnetoelectric (ME) composites based on observed giant magnetoelectric effects, rich physics, and potential for real-world applications, such as ultrasensitive roomtemperature magnetic field sensors for biomedical fields, actuators, energy harvesters, and nonlinear frequency mixing devices [1-7]. Magnetoelectric composites are actively being investigated along other room-temperature magnetometry approaches, such as magnetoresistance and atomic spin polarization. The magnetoelectric effect in composites is mediated via strain coupling between phases of different ferroic ordering, i.e., piezomagnetism and piezoelectricity and is orders of magnitude greater than in single-phase materials $[1,3,8-14,17,18]$. This has motivated numerous investigations of piezoelectric and magnetostrictive materials and interaction effects at different scales ranging

*makr@tf.uni-kiel.de

Published by the American Physical Society under the terms of the Creative Commons Attribution 4.0 International license. Further distribution of this work must maintain attribution to the author(s) and the published article's title, journal citation, and DOI. from macroscopic to nanoscale magnetoelectric composites. Significant research activity also takes place in the related field of magnetoelectric multiferroics, e.g., engineered materials down to the atomic-layer scale with more than one order parameter per phase and different effects yielding magnetoelectric coupling [5,7]. The strainmediated magnetoelectric effect in composites consisting of piezomagnetic and piezoelectric materials is a secondorder product-property effect and involves the physics of magnetism, magnetoelasticity, piezo or ferroelectricity, and strain coupling at different scales. Composite structures of different dimensionalities have been investigated, i.e., $0 \mathrm{D}, 1 \mathrm{D}, 2 \mathrm{D}$, and $3 \mathrm{D}$, requiring specific orientations of the ferroic ordering in the different phases and corresponding excitation modes. Of the different possible composite structures, layered composites have received the most attention as sample production can take advantage of existing and reproducible thin-film deposition and MEMS microfabrication techniques [9-12,14,17,18]. Furthermore, resonance-enhanced magnetoelectric coupling has been observed in bending mode of magnetoelectriccomposite cantilevers, giving rise to giant ME effects [14], albeit narrowing the bandwidth to the linewidth of the resonance peak. This has resulted in room-temperature magnetic field sensors of sub-picotesla detection limits, i.e., almost sufficient for detection of biomagnetic fields in the 
$100 \mathrm{fT} / \mathrm{Hz}^{1 / 2}$ range originating from brain currents. Magnetoencephalography (MEG) systems use magnetic field data from hundreds of superconducting quantum interference device (SQUID) sensors placed around the cortex to calculate the neural current source distribution $[19,20]$ thus not requiring the small sensor sizes of scanning microscopy. However, there are many applications, e.g., deep brain stimulation [21,22], where SQUID MEG systems are not compatible with the clinical environment due to shielding requirements or system size disallowing any motion. Cantilevers have enabled detection of numerous small force, surface, and interface effects with and without investigation of the corresponding fundamental limits, e.g., Stove et al. [23], Li et al. [24], and Finot et al. [25]. For magnetoelectric cantilevers, resonance-enhanced excitation augments the many interactions already involved by the issues of multiple quality-factor-limiting loss mechanisms. Magnetoelectric composites may be excited in different excitation modes. Specifically, bending modes are of interest for achieving large resonance-enhanced magnetoelectric effects at the low frequencies required for detection of biomagnetic signals. Torsion and longitudinal modes have orders of magnitude greater resonance frequencies than bending modes for the cantilevers of length and width $\gg$ thickness investigated here, and their excitation by low-frequency biomagnetic fields is expected to be very small due to frequency mismatch even if allowed by low device symmetry or nonuniform fields. Modulation techniques for detection of low-frequency biomagnetic signals have been investigated based on the nonlinearity of ferroic materials with signals appearing as sidebands to a carrier $[15,16]$ and increasing the bandwidth. These are not considered here. This paper focuses on the direct $\mathrm{ME}$ effect.

The scope of the resonance-enhanced magnetoelectriccomposite problem also requires the investigation of noise issues for understanding the ultimate limits of sensors and other devices. Here, noise sources intrinsic to individual ferroic phases, as well as those attributable to the composite device and signal amplifier, require consideration, in particular, for magnetic field sensors operating at room temperature [27-33] as a potential alternative to SQUID magnetometry of biomagnetic fields.

Previously, theoretical models for the transverse magnetoelectric effect of layered composites in bending mode were derived including the results for the ME response of multilayer composites of different layer sequences for resonant and static magnetic field excitation [34-40]. In addition, the bending-mode ME response for openand short-circuit operation of the piezoelectric phase in magnetoelectric-composite cantilevers in the resonant and static cases were given. These models are based on linear constitutive equations coupling the local fields of strain and stress with the magnetic, electric, and electric displacement fields via bulk material properties, whereby linear behavior in the inherently nonlinear ferroic materials refers to small field variations at the operating point.

For calculation of the ME response of cantilevers in bending mode in the resonance-enhanced case, the constitutive equations are solved in conjunction with the Euler-Bernoulli beam theory using bending mode boundary conditions and assuming negligible strain relaxation at the layer interfaces.

Investigations of intrinsic noise in magnetoelectric composites for magnetic field sensing are presented for direct detection and modulation techniques. Results are largely based on analyzing the Johnson-Nyquist noise produced via the fluctuation-dissipation theorem [26] from dielectric losses in the piezoelectric phase and calculating the corresponding equivalent magnetic field fluctuations [2730,32,33]. Xing et al. [27] investigated the frequency dependence of the signal-to-noise ratio of a laminated MEcomposite preamplifier system for nonresonant direct magnetoelectric detection using an equivalent circuit model fitted to experimental data from a ME laminate sensor and finding a $1 / f$ dependence of the detection limit at low frequency. Zhuang et al. [32] investigated the noise behavior for several field modulation techniques and attributed the dominant noise to mechanical losses in the laminated composite or instrumentation electronics with thermalmechanical fluctuations not being dominant. Jahns et al. $[30,31]$ studied the effect of Johnson-Nyquist noise from dielectric losses in the piezoelectric phase of the composite as well as amplifier-intrinsic noise sources for resonant ME-composite cantilevers, comparing the findings to experimental results. However, thermal vibrations were neglected based on estimated amplitudes of a few picometers [30] and no systematic investigation of the noise-model behavior in conjunction with the corresponding behavior of a ME-response model was performed in the above noise investigations. Furthermore, the effect of thermal-vibration noise [41-43] on magnetoelectric detection was not evaluated by the above authors. Durdaut et al. [45] investigated thermal-vibration noise in a ME composite and identified it as the dominant noise source for the analyzed cantilever. The issue of thermal-vibration noise in cantilevers emerged around atomic force microscopy (AFM) and was also identified as an important noise source in MEMS [41-43]. It is based on the equipartition theorem yielding the expectation value of thermally excited vibration energy of $(1 / 2) k_{B} T$ for every mode of a ME cantilever, $k_{B}$ denoting the Boltzmann constant and $T$ the temperature. In resonance-enhanced magnetoelectric composite cantilevers, thermal energy and the detected magnetic field excite the same mode. However, a combined theory of the ME-response and noise models, including JohnsonNyquist noise and thermal-vibration noise in magnetoelectric composite cantilevers, has not been presented. This is essential for identification of dominating noise sources and a systematic investigation of the resulting fundamental 
versus nonfundamental magnetic field detection limits. To address this issue, we present a combined analytic theory for the ME-response and ME-sensor noise components from thermal vibrations, Johnson-Nyquist noise from dielectric losses, and amplifier-intrinsic noise sources to yield the magnetic field detection limit of magnetoelectriccomposite cantilevers. The magnetoelectric response and noise components in composites depend on the excited mode and dimensional and material parameters. Results of the theory for the first bending mode are presented using the well-researched system $\left(\mathrm{Fe}_{90} \mathrm{Co}_{10}\right)_{78} \mathrm{Si}_{12} \mathrm{~B}_{10} / \mathrm{Si} / \mathrm{AlN}$ for the magnetostrictive, substrate, and piezoelectric layers, respectively, whereby the material choices are motivated as follows. $\left(\mathrm{Fe}_{90} \mathrm{Co}_{10}\right)_{78} \mathrm{Si}_{12} \mathrm{~B}_{10}$ with stoichiometry $\left(\mathrm{Fe}_{90} \mathrm{Co}_{10}\right)_{78} \mathrm{Si}_{12} \mathrm{~B}_{10}$ [12] is an amorphous soft magnetic metal with piezomagnetic constant exceeding that of $\mathrm{Tb}_{x} \mathrm{Dy}_{1-x} \mathrm{Fe}_{2}(x=0.3)$ (Terfenol-D). AlN is hexagonal with about 3 times greater piezoelectric constant and about 300 times smaller dielectric constant than $\mathrm{Pb}\left(\mathrm{Zr}_{0.52} \mathrm{Ti}_{0.48}\right) \mathrm{O}_{3}$ (PZT). In addition, AlN has small dielectric losses [46,47], which is important for JohnsonNyquist noise considerations. The layer sequence with the $\mathrm{Si}$ substrate in the center is motivated by greater ME response [37,39] and processing advantages for the functional layers, i.e., $\left(\mathrm{Fe}_{90} \mathrm{Co}_{10}\right)_{78} \mathrm{Si}_{12} \mathrm{~B}_{10}$ and $\mathrm{AlN}$, due to low surface roughness on both sides of the $\mathrm{Si}$ substrate. Using this theory, we systematically investigate a wide range of ME cantilever sizes and layer thicknesses, calculating magnetic-field-induced ME-response signals and individual and sums of uncorrelated noise signals at the output of matched charge amplifiers including thermal vibration, Johnson-Nyquist, amplifier, and cable-associated noise and filter effects, to yield the detection limit behavior for given resonance frequencies and quality factors. For $\left(\mathrm{Fe}_{90} \mathrm{Co}_{10}\right)_{78} \mathrm{Si}_{12} \mathrm{~B}_{10} / \mathrm{Si} / \mathrm{AlN}$ composites, we identify thermal vibrations as the dominating noise source for most cantilever dimensions and determine parametrized fundamental limits to the achievable magnetic field detection limit. As a result of the dominating thermal-vibration noise, other nonfundamental noise sources and the engineering issues associated with them [48] largely do not affect the findings of this paper. To investigate the limits of ultrasensitive ME composites, we systematically address scaling issues of the thermalvibration-noise-limited magnetic field detection limit with cantilever size, layer thicknesses, resonance frequency, and quality factor. Throughout this work cantilever size refers to the investigation to cantilevers of constant lengthto-width (aspect) ratio of 3. Constant quality factors are used, excluding the dimensional scaling behavior of different damping mechanisms, e.g., viscous-air damping [49], thermoelastic damping [50], support losses [51], eddy current, and other intrinsic losses in the magnetostrictive layers [52,53], investigations of which for magnetoelectric composites will be given elsewhere.
The paper is organized as follows. We present the model details for the ME-response theory, the noise model including different noise components, and the magnetic field detection limit in Secs. II, III, and IV, respectively. In Sec. V, results are presented and discussed, starting with the frequency, sensitivity, noise and detection limit behavior of constant-size cantilevers, and corresponding results for size-scaled cantilevers of constant resonance frequency. Further investigation addresses the behavior with functional layer thickness and detection limit scaling with cantilever length, resonance frequency, and quality factor.

\section{ME RESPONSE}

Figure 1(a) shows the $\left(\mathrm{Fe}_{90} \mathrm{Co}_{10}\right)_{78} \mathrm{Si}_{12} \mathrm{~B}_{10} / \mathrm{Si} / \mathrm{AlN}$ composite cantilever consisting of $\left(\mathrm{Fe}_{90} \mathrm{Co}_{10}\right)_{78} \mathrm{Si}_{12} \mathrm{~B}_{10}$ and AlN layers on opposite sides of the Si substrate. Also shown is the coordinate system with cantilever length along $x$ and bending displacement along $z$ directions. Excitation of the cantilever bending mode by resonantly oscillating magnetic fields produces piezoelectric
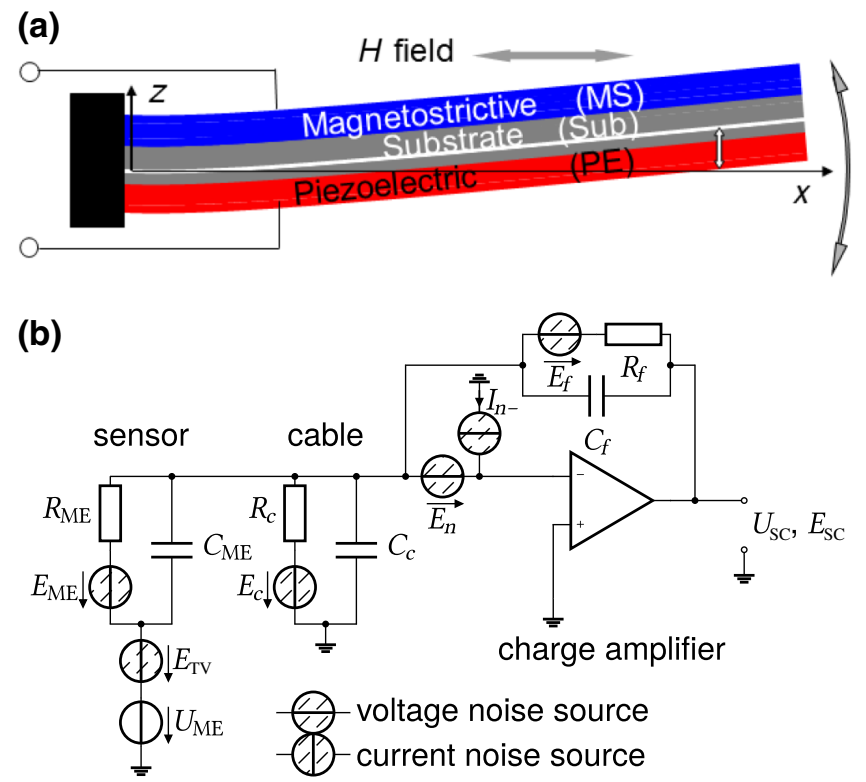

FIG. 1. Magnetoelectric-composite cantilever of straincoupled magnetostrictive, substrate, and piezoelectric layers, including the coordinate system with $z$ originating at the neutral plane (white line). Oscillating magnetic fields in the $x$ direction as well as $(1 / 2) k_{B} T$ thermal vibrations at the resonance frequency excite the lowest order bending mode, polarizing the piezoelectric layer and producing charges at the leads (a). Equivalent circuit of the ME cantilever sensor connected to a charge amplifier. Bending strain-induced piezoelectric signals and thermal-vibration noise with Johnson-Nyquist noise from dielectric losses of the piezoelectric layer and cable as well as noise from the charge amplifier and feedback loop yield an overall signal-to-noise ratio and detection limit $(\delta H)(b)$. 
charges via strain coupling, whereby the elastic straincoupled theory of the nanocomposite assumes thicknessindependent material parameters and ideal interface coupling without strain relaxation. The strain-induced charges of the AlN layer are collected by contacts over the full area of cantilever with length $a$ and width $b$. The strain in $x$ direction is described for each layer as a function of stress and external fields by linear constitutive equations (1)-(4). The piezoelectric and piezomagnetic effects are nonlinear for large field amplitudes. The linearity refers to small field variations near the operating points.

$$
\begin{aligned}
S_{3 m} & =s_{33 m} T_{3 m}+d_{33 m} H_{3} \\
S_{1 s} & =s_{11 s} T_{1 s} \\
S_{1 p} & =s_{11 p} T_{1 p}+g_{31 p} D_{3} \\
E_{3} & =-g_{31 p} T_{1 p}+\beta_{33} D_{3} .
\end{aligned}
$$

Equation (1) refers to the magnetostrictive (MS) layer, Eq. (2) to the substrate, and Eqs. (3) and (4) to the piezoelectric (PE) layer. $S$ and $T$ denote the strain and stress and $D, H$, and $E$ the electric displacement, magnetic and electric fields, respectively. The elastic compliances are given by $s$ with subscripts $m, p$, and $s$ denoting the MS, $\mathrm{PE}$, and substrate phase, respectively. The piezomagnetic constant is denoted $d_{33 m}$ and $g_{31 p}$ refers to the transverse piezoelectric and $\beta_{33}$ to the inverse dielectric constant. The orientations are given in local coordinates with subscript 3 denoting $x$ for the MS layer, while for the PE and Sub layers 1 denotes $x$ and 3 denotes $z$ (Fig. 1).

The calculation of the resonant ME response [39] (Appendix A) involves simultaneously solving the above constitutive equations and the equation of motion for the bound-free cantilever bending mode in the EulerBernoulli beam theory approximation, whereby conservation of stress is employed and the strain varies linearly with $z$ coordinate across the cantilever. In addition, shortcircuit operation is induced by the charge amplifier. For the resonance-enhanced bending motion, the ME response is dominated by bending strains inside the cantilever which originate from inertia. The field-induced strains exciting the oscillation are smaller for sufficiently high quality factors and can be neglected when compared to the bending strains in the derivation of the resonant ME response.

The resonant short-circuit ME response $\alpha$ normalized by the quality factor is defined as

$$
\frac{\alpha_{\mathrm{ME}-\mathrm{SC}}}{Q_{f}}=\frac{1}{Q_{f}} \frac{1}{h_{m}+h_{p}} \frac{d Q}{d H_{3}}
$$

Here, $h_{m}, h_{p}$, and $Q_{f}$ denote the layer thicknesses of the magnetostrictive and piezoelectric layers and the quality factor of the cantilever, respectively. Short-circuit operation is induced by the charge amplifier.
The resonant short-circuit ME response, defined as the ratio of piezoelectric charge $Q$ to the inducing $H$ field normalized by the quality factor $Q_{f}$ and the functional layer thicknesses $h_{m}$ (MS) and $h_{p}$ (PE), was derived previously with the result [39] (Appendix A)

$$
\frac{d Q}{d H}=\frac{T_{1} T_{6} b[\cosh (k a) \sin (k a)+\sinh (k a) \cos (k a)]}{T_{7} k[1+\cosh (k a) \cos (k a)]} .
$$

Normalization by the quality factor $Q_{f}$ excludes resonance enhancement details, loss mechanisms, and damping effects from the ME coefficients [40]. Here, the mode parameter $k$ equals $1.875 / a$ for the first mode, and the terms $T_{1}, T_{6}$, and $T_{7}$ (not to be confused with the stress $T$ ) are given in Appendix A. The derived terms are given for the magnetostrictive-substrate-piezoelectric (MSP) layer sequence of Fig. 1(a). However, the theory is easily applied to the magnetostrictive-piezoelectricsubstrate or substrate-magnetostrictive-piezoelectric layer sequences by replacing the corresponding terms in the ME response and the thermal-vibration noise expression (10) given in Sec. III and inserting the corresponding result for the neutral plane [39].

The sensitivity $S$ at the charge amplifier output in V/Oe is given as

$$
S=\frac{\alpha_{\mathrm{ME}-\mathrm{SC}}\left(h_{m}+h_{p}\right)}{C_{f}} .
$$

whereby the gain is determined by the ratio of the sensor to the feedback capacitance, i.e., $C_{\mathrm{ME}} / C_{f}$. Throughout this work $C_{f}$ is calculated to yield a gain of 10 . As the gain affects sensitivity and noise, there is no net effect on the detection limit results.

\section{NOISE MODEL}

Figure 1(b) shows the magnetoelectric composite connected to a charge amplifier circuit including the equivalent circuit for the ME sensor and cable. All capacitances are subject to dielectric losses and thus produce Johnson-Nyquist noise based on the fluctuation-dissipation theorem. For the ME sensor, the AlN layer of capacitance $C_{\mathrm{ME}}$ (full-area electrodes assumed here) produces dielectric losses $R_{\mathrm{ME}}$, giving rise to the noise voltage $E_{\mathrm{ME}}$. Corresponding capacitances, dielectric losses, and noise voltages for the cable and feedback capacitance are denoted $C_{c}, R_{c}, E_{c}$ and $C_{f}, R_{f}$, and $E_{f}$, respectively.

Additional noise sources are the thermal-vibration noise $E_{\mathrm{TV}}$ [41-43] produced by the $(1 / 2) k_{B} T$ expectation value of thermal vibration excitation for each mode and amplifier-intrinsic voltage and current noise $E_{n}$ and $I_{n}$, respectively. The result of adding the uncorrelated noise sources, including filter effects yielding the total noise density at the charge amplifier output, was given by Jahns [31] as 


$$
\begin{gathered}
V_{\text {noise-SC }}=\left[\left(\frac{\left|Z_{f}\right|}{\left|Z_{\mathrm{ME}}\right|}\right)^{2}\left|\frac{1}{1+i 2 \pi f C_{\mathrm{ME}} R_{\mathrm{ME}}}\right|^{2} \frac{E_{\mathrm{ME}}{ }^{2}}{\Delta f}\right. \\
+\left(\frac{\left|Z_{f}\right|}{\left|Z_{\mathrm{ME}}\right|}\right)^{2} \frac{E_{\mathrm{TV}}{ }^{2}}{\Delta f}+\left|1+\frac{Z_{f}}{Z_{\mathrm{MEC}}}\right|^{2} E_{n}{ }^{2}+\left|Z_{f}\right|^{2} I_{n}{ }^{2} \\
\left.+\left|\frac{1}{1+i 2 \pi f C_{f} R_{f}}\right|^{2} \frac{E_{f}{ }^{2}}{\Delta f}+\left|Z_{f}\right|^{2} \frac{E_{c}{ }^{2}}{R_{c}{ }^{2} \Delta f}\right]^{1 / 2}
\end{gathered}
$$

Here, $f$ is the cantilever resonance frequency, $\Delta f$ is the noise bandwidth and $Z_{\mathrm{ME}}, Z_{f}$, and $Z_{\mathrm{MEC}}$ refer to various impedances detailed in Appendix B. The first term denotes the sensor noise, i.e., the noise density from the dielectric losses in the AlN layer. The second term gives the noise density from thermal vibrations of the cantilever. The remaining four terms give the noise densities from the voltage and current noises of the operational amplifier, the feedback capacitance, and the cantilever-amplifier cable, respectively. Details of Eq. (8), i.e., dielectric losses and impedances $Z$, are given in Appendix B and noise model parameters in Table II, including quality factor and bandwidth effects. The signal voltage $U_{\mathrm{ME}}$ [Fig. 1(b)] is produced by magnetic-field-induced cantilever bending at the sensor and corresponds to the amplifier output signal calculated via the ME coefficient.

Calculating the thermal-vibration noise $E_{\mathrm{TV}}$ of the ME cantilever involves determining the thermal vibration amplitude at the cantilever tip [43] for the ME cantilever and calculating the corresponding ME sensor signal produced by cantilever bending from thermal excitations. The expectation value of the peak thermal amplitude $w_{\mathrm{TV}}$ in the $z$ direction at the tip of an AFM cantilever was calculated by Salapaka et al. [43] for a single mode $j$ as

$w_{\mathrm{TV}}=\left[\frac{k_{B} T c_{j} \phi_{j}{ }^{2}}{\pi} \int_{\omega_{j}-\Delta f \pi}^{\omega_{j}+\Delta f \pi} \frac{1}{\left(k_{j}-m_{j} \omega_{j}^{2}\right)^{2}+c_{j}{ }^{2} \omega_{j}^{2}} d \omega\right]^{1 / 2}$,

and depends on the quality factor $Q_{f}$ and the noise bandwidth $\Delta f$, i.e., the full width centered at the resonance frequency $\omega_{j}$ here. Here, $k_{j}$ is the mode-dependent force constant, $m_{j}$ the modal mass, $\omega_{j}=\left(k_{j} / m_{j}\right)^{0.5}$ the resonance frequency, $\phi_{j}=-8.27$, and $c_{j}=\left(k_{j} m_{j}\right)^{0.5} Q_{f}^{-1}$ for the first mode $j=1$.

Throughout this work, we consider the case where the noise bandwidth is much smaller than the $Q_{f}$-determined halfwidth of the ME-response and thermal vibration mode.

For the ME cantilever, the corresponding thermalvibration-induced ME-sensor signal is arrived at by calculating the thermal-noise-equivalent charge $w_{\mathrm{TV}}(d Q / d H)$ $(d H / d z)$ via the magnetic-field-induced charge term $d Q / d H$ from the ME response and the thermal noise equivalent magnetic field $w_{\mathrm{TV}}(d H / d z)$, i.e., the field $H$ which produces a cantilever bending in the $z$ direction of $w_{\mathrm{TV}}$, and dividing by the sensor capacity $C_{\mathrm{ME}}$ [30]. This yields

$$
E_{\mathrm{TV}}=\frac{w_{\mathrm{TV}}}{C_{\mathrm{ME}}} \frac{d Q}{d H} \frac{d H}{d z}
$$

with

$$
\frac{d Q}{d H} \frac{d H}{d z}=\frac{-T_{1} b k[\cosh (k a) \sin (k a)+\sinh (k a) \cos (k a)]}{\sin (k a) \sinh (k a)} .
$$

Details of the derivation and parametrized evaluation of Eqs. (9)-(11) for the ME-composite cantilever are given in Appendix C.

\section{DETECTION LIMIT}

We define the detection limit $(\delta H)$ as the ratio of noise density and sensitivity, i.e., the magnetic field for a signalto-noise ratio of one. This is given as

$$
\delta H=\frac{V_{\text {noise-SC }}}{S}
$$

for small noise bandwidths compared to the linewidth of the resonant bending mode. Essentially, all model parameters affect the detection limit, i.e., material, dimensional, modal, and amplifier electronics parameters. Our analytic model has no fitting parameters. In this work, we only consider results for the first bound-free bending mode using the strain-induced charges of the full area of the 3-layer magnetoelectric cantilever. Extension of the theory to higher modes is straight forward but requires lengthy extensions to account for the altered strain distributions along the cantilever. Investigation of higher-order bending modes yields similar scaling laws of the detection limit with cantilever size [44]. However, torsion modes of thin long cantilevers have much greater resonance frequencies and different dimensional scaling laws of the resonance frequency than bending modes, suggesting that detection limit scaling laws expected of corresponding models involving constitutive equations, resonant $\mathrm{ME}$ response, and thermal-noise-limited behavior may also be different. In the bending-mode model here, effects from additional thin layers present in real devices, e.g., contact, adhesion, and seed layers, are neglected. We focus on the main effects of the detection limit behavior in this large parameter space and exclude loss mechanisms by using constant quality factors. We also neglect microscale structural, dielectric, and magnetic effects by using layer-thickness-independent experimental material parameters and do not consider demagnetization field effects, the latter of which will be treated elsewhere. 


\section{RESULTS AND DISCUSSION}

For systematic analysis of the effects of cantilever dimensions and layer thicknesses on resonance frequency, ME response, sensitivity, different noise components, and the magnetic field detection limit, we proceed by focusing on scaling behavior, moving from many to a few constrained parameters. Following this outline, Figs. 2, 3,6 , and 7 give results for cantilevers of constant functional layer thicknesses, whereby Fig. 2 shows findings for constant size and varying resonance frequency and Fig. 3 for variable size scaled to yield a constant resonance frequency. Figure 4 displays corresponding results for arbitrary ratios of functional layer thicknesses and Fig. 5 the behavior with functional layer thickness. Figures $6-8$ show cantilever size scaling results of detection limits at constant resonance frequency for fixed (Fig. 6), different (Fig. 7), and proportionally scaled functional layer thicknesses (Fig. 8). The aspect ratio of cantilever length to width is kept constant $(=3)$ throughout this work.

Figure 2 details the behavior with $\mathrm{Si}$ substrate thickness $\left(h_{s}=0.5 \ldots 50 \mu \mathrm{m}\right)$ for cantilevers of $3 \mathrm{~mm}$ length, $1 \mathrm{~mm}$ width, functional layers of $0.2 \mu \mathrm{m}$ AlN, $1.8 \mu \mathrm{m}$ $\left(\mathrm{Fe}_{90} \mathrm{Co}_{10}\right)_{78} \mathrm{Si}_{12} \mathrm{~B}_{10}$, and constant quality factor $Q_{f}=$ 1000. The resonance frequency [Fig. 2(a)] varies from 0.2 to $8 \mathrm{kHz}$. The short-circuit ME coefficient (normalized to $Q_{f}=1$ ) reaches a maximum between 1 and $2 \mu \mathrm{m}$ substrate thickness where magnetic field excitation, neutral plane position, and strain coupling into the AlN layer produce the greatest ME response [39] [Fig. 2(b)]. For thick substrates it falls off, reaching a slope nearly proportional to $h_{s}^{-1}$. The sensitivity behavior (at the charge amplifier output for $Q_{f}=1000$ and gain $=10$ ) follows the ME coefficient and the feedback capacity (= sensor capacity/gain) is constant [Fig. 2(b)]. The behavior of noise contributions with substrate thickness $h_{s}$ is given in Fig. 2(c), with thermal-vibration noise dominating (maximum slope $\propto h_{s}^{-1}$ at $h_{s}=50 \mu \mathrm{m}$ ), smaller amplifier and cable noise (vanishing slope at $h_{s}=50 \mu \mathrm{m}$ ) and sensor noise from dielectric fluctuations in the AlN layer (maximum slope $\propto h_{s}^{-1 / 2}$ ). The slopes are attributed to the frequency dependence of the noise components and in particular to the vibration-energy behavior of the cantilevers versus the constant $(1 / 2) k_{B} T$ energy and lowpass filter effects in the sensor-amplifier circuit. It should be pointed out that the quality factor affects the sensitivity and thermal-vibration noise but not the sensor and amplifier-cable noise as will be discussed in conjunction with Fig. 6. Figure 2(d) displays the behavior of the detection limit ( $\propto$ noise/sensitivity). The lowest detection limit of about $1 \mathrm{pT} / \mathrm{Hz}^{1 / 2}$ is calculated for a substrate thickness near $h_{s}=25 \mu \mathrm{m}$, i.e., greatly differing from the sensitivity and ME-response maximum.

Materials properties of the $\left(\mathrm{Fe}_{90} \mathrm{Co}_{10}\right)_{78} \mathrm{Si}_{12} \mathrm{~B}_{10} / \mathrm{Si} / \mathrm{AlN}$ composite affect the results of Fig. 2 as follows. $T_{1}$

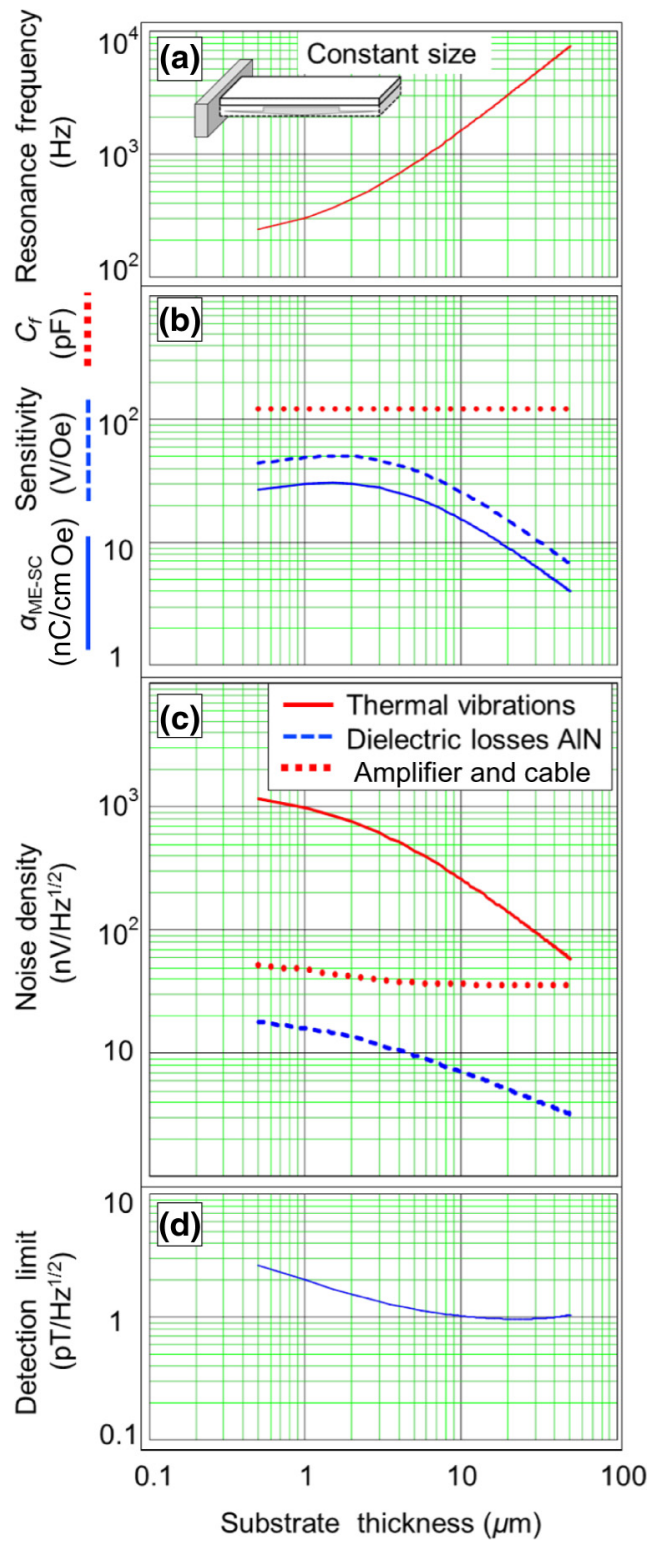

FIG. 2. Resonance frequency (a), ME response, sensitivity, and feedback capacitance (b), noise densities at amplifier output from thermal vibrations, dielectric losses of the AlN layer, and amplifier and cable noises (c), and resulting detection limit for resonantly exciting magnetic fields (d) for a $\left(\mathrm{Fe}_{90} \mathrm{Co}_{10}\right)_{78} \mathrm{Si}_{12} \mathrm{~B}_{10} / \mathrm{Si} / \mathrm{AlN}$ cantilever as a function of $\mathrm{Si}$ substrate thickness. Cantilever size of $3 \mathrm{~mm}$ length, $1 \mathrm{~mm}$ width, layer thicknesses of $1.8 \mu \mathrm{m}\left(\mathrm{Fe}_{90} \mathrm{Co}_{10}\right)_{78} \mathrm{Si}_{12} \mathrm{~B}_{10}$ and $0.2 \mu \mathrm{m}$ AlN, and quality factor $Q_{f}=1000$ are constant. In Fig. 2(b), the ME response [39] is normalized to $Q_{f}=1$ and the sensitivity (for $Q_{f}=1000$ ) refers to the charge amplifier output for a gain of 10. Spectral-noise densities depend strongly on resonance frequency and show thermal vibrations dominating and smaller contributions from amplifier, cable, and dielectric losses of the AlN layer [Fig. 2(c)]. The behavior of sensitivity (b) and thermalvibrations-dominated noise (c) yield a minimum of the detection limit below $1 \mathrm{pT} / \mathrm{Hz}^{1 / 2}$ near $25 \mu \mathrm{m} \mathrm{Si}$ at about $f=4 \mathrm{kHz}$, i.e., greatly different from the ME-response maximum near $2 \mu \mathrm{m} \mathrm{Si}$ and $f=400 \mathrm{~Hz}$. 

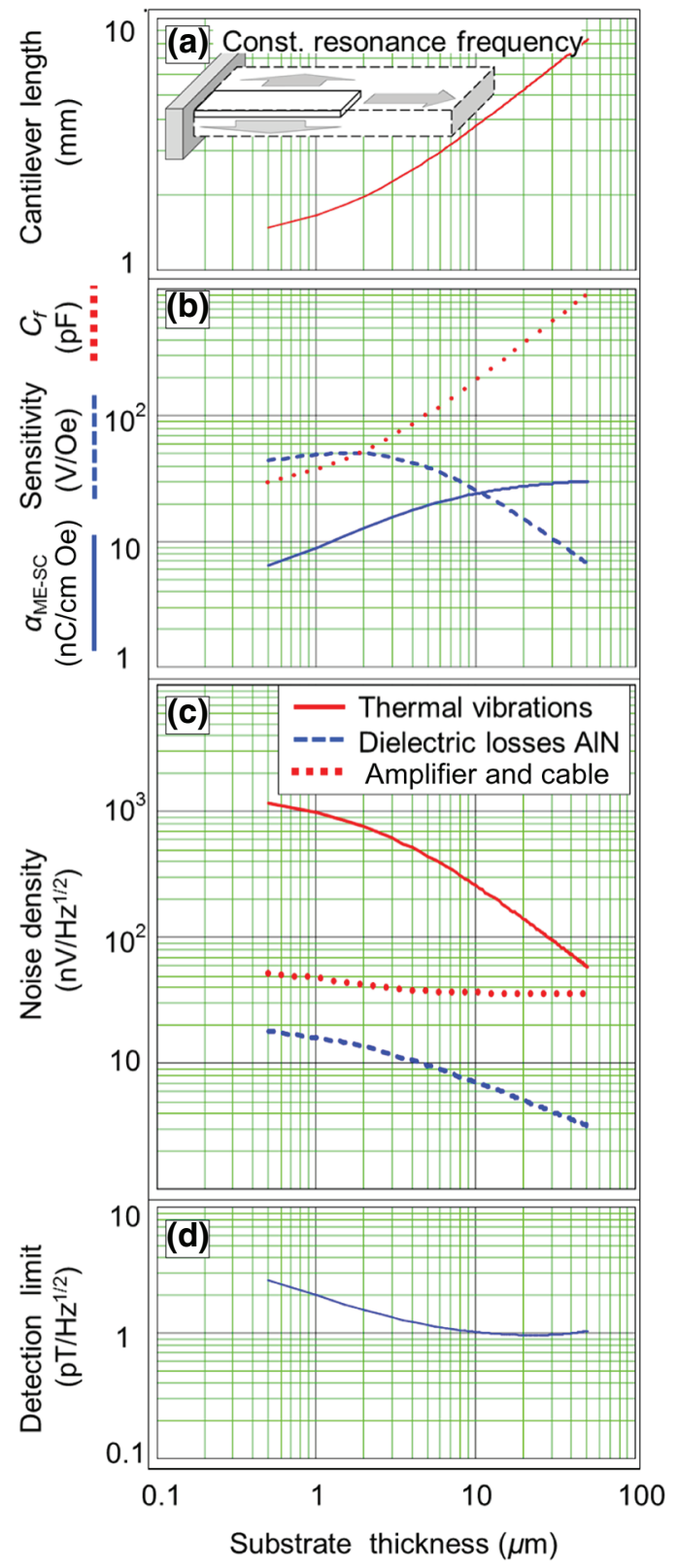

FIG. 3. ME response, sensitivity, noise densities, and detection limit behavior as in Fig. 2; however, for length and width-scaled cantilevers yielding a constant $1 \mathrm{kHz}$ resonance frequency. Quality factor $Q_{f}=1000,\left(\mathrm{Fe}_{90} \mathrm{Co}_{10}\right)_{78} \mathrm{Si}_{12} \mathrm{~B}_{10}$ and AlN layer thicknesses, and 3:1 aspect ratio are held constant. The size-scaled $f=1 \mathrm{kHz}$ cantilevers yield different ME response and feedback capacitance but identical sensitivity behavior (gain =10) (b), noise density components (c), and detection limit behavior (d) as the unscaled cantilevers of Fig. 2.

(A4) is proportional to the piezoelectric constant $g_{31 p}$ and $T_{6}$ (A8) to the piezomagnetic constant $d_{33 m}$ resulting in the ME response (6) [Fig. 2(b)] being proportional to the product $g_{31 p} d_{33 m}$. All terms of the noise density (8) as well as the thermal vibration amplitude $w_{\mathrm{TV}}$ and $(d Q / d H)(d H / d z)(11)$ are independent of $d_{33 m}$. The terms

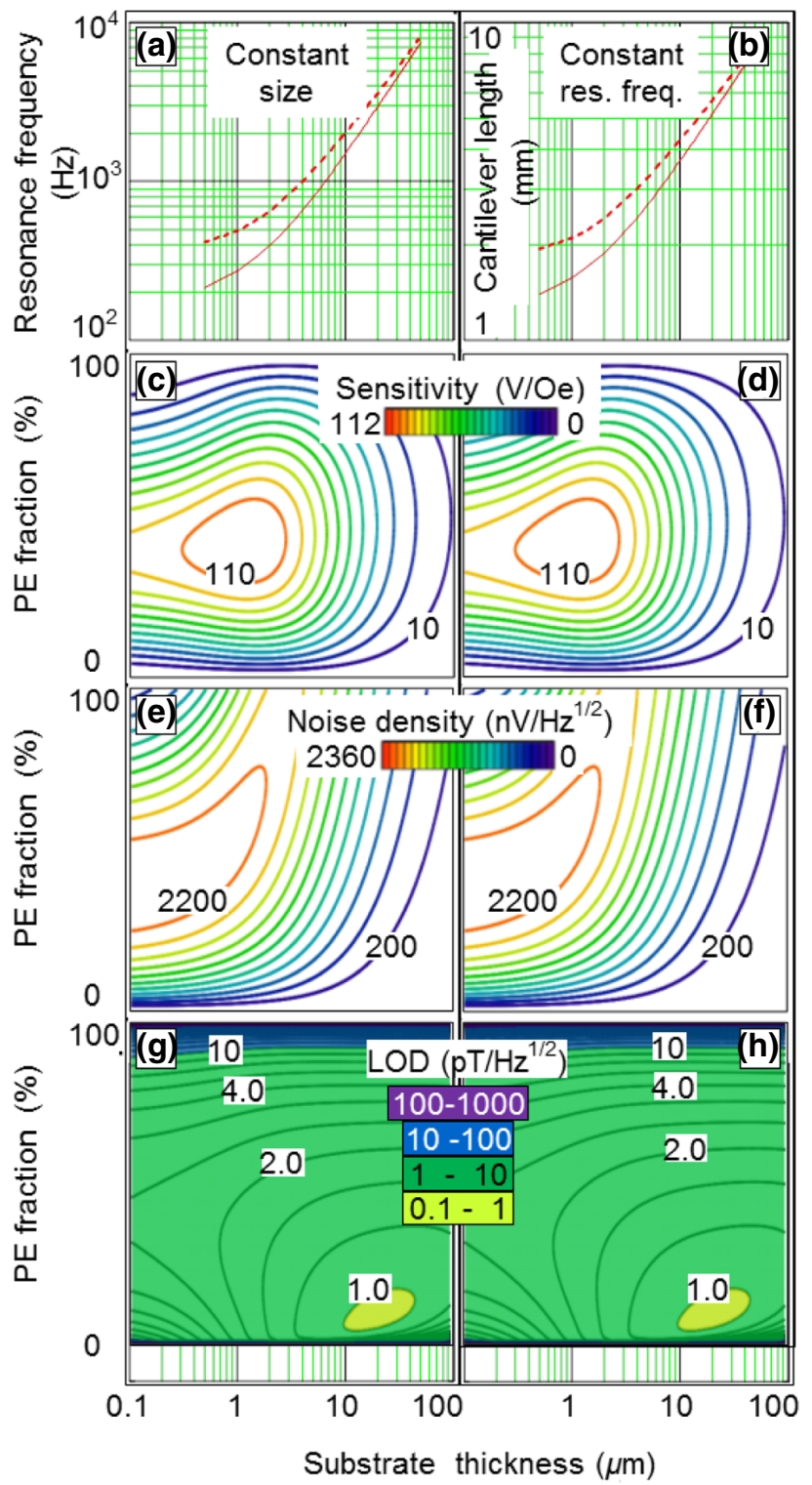

FIG. 4. Behavior of sensitivity, total noise density, and detection limit with substrate thickness and functional-layer-thickness ratio (PE fraction defined as $\left.h_{p} /\left(h_{p}+h_{m}\right)\right)$ for constant $\left(h_{p}+\right.$ $\left.h_{m}=2 \mu \mathrm{m}\right)$ functional layers. Results for constant $3 \times 1 \mathrm{~mm}^{2}$ size cantilevers [Figs. 4(c), 4(e) and 4(g)] are compared to those for constant $1 \mathrm{kHz}$ resonance frequency cantilevers [Figs. 4(d), 4(f) and 4(h)] produced by size scaling for constant 3:1 aspect ratio and quality factor $Q_{f}=1000$. Figures 4(a) and 4(b) give the frequency and length results for $\mathrm{PE}=0 \%$ (solid line) and $100 \%$ (ellipses), respectively. Sensitivity [Figs. 4(c) and 4(d)] and noise densities [Figs. 4(e) and 4(f)] at amplifier output $($ gain $=10)$ are given on linear scales with $10 \mathrm{~V} / \mathrm{Oe}$ and $200 \mathrm{nV} / \mathrm{Hz}^{1 / 2}$ contour line spacing, respectively. Detection limits are given on a logarithmic scale with $1 \mathrm{~dB}$ contour lines. ME response, noise density, and detection limit behavior differ greatly. However, the behavior of corresponding constant-size and size-scaledconstant-frequency ME cantilevers is identical. The lowest detection limit is found near $10 \% \mathrm{PE}$ fraction and $h_{s}=25 \mu \mathrm{m} \mathrm{Si}$ substrates, i.e., greatly differing from the sensitivity maximum but near the noise minimum. 


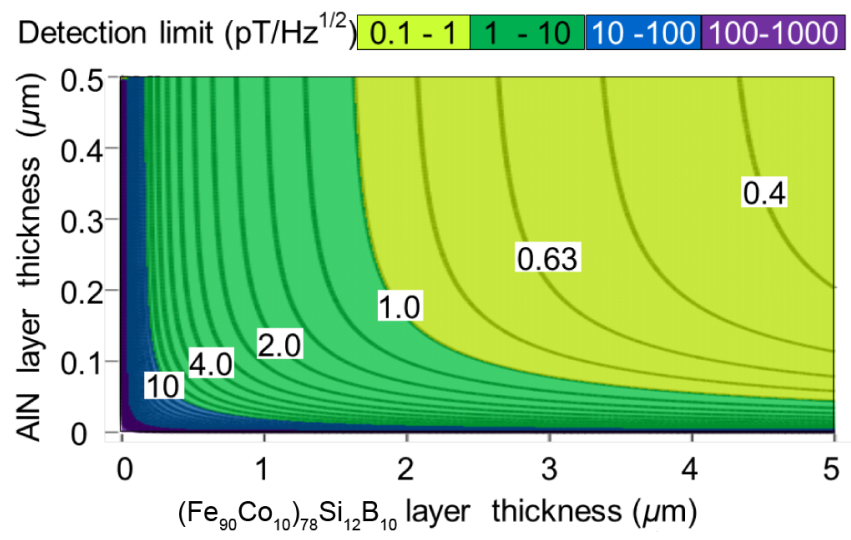

FIG. 5. Resonant magnetic field detection limit behavior of 3 -mm-long and $1-$ mm-wide $\left(\mathrm{Fe}_{90} \mathrm{Co}_{10}\right)_{78} \mathrm{Si}_{12} \mathrm{~B}_{10} / \mathrm{Si} / \mathrm{AlN}$ cantilevers with $\left(\mathrm{Fe}_{90} \mathrm{Co}_{10}\right)_{78} \mathrm{Si}_{12} \mathrm{~B}_{10}$ and AlN layer thickness for constant $50 \mu \mathrm{m}$ Si substrate $\left(Q_{f}=1000, f \sim 7.5-7.8 \mathrm{kHz}\right)$. Results are given on a logarithmic scale with $1 \mathrm{~dB}$ contour lines and show a strong dependence on MS layer thickness and only small changes with PE layer thickness if the former exceeds the latter by about $10 \times$.

for amplifier and cable noise, sensor noise, and for the thermal vibration amplitude $w_{\mathrm{TV}}(9)$ are independent of $g_{31 p} .(d Q / d H)(d H / d z)(11)$ is proportional to $g_{31 p}$, making the thermal-vibration noise term in Eq. (8) proportional

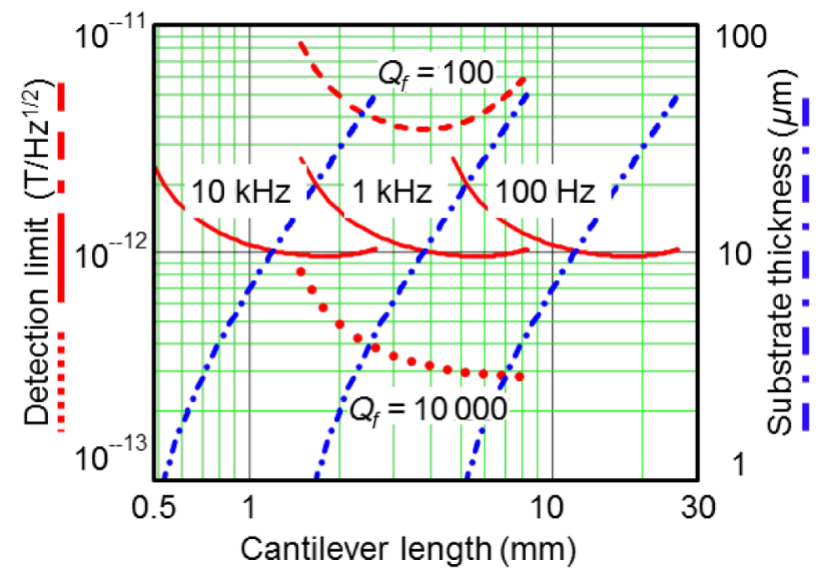

FIG. 6. Detection limit scaling with cantilever size (aspect ratio 3) for constant $1.8 \mu \mathrm{m}\left(\mathrm{Fe}_{90} \mathrm{Co}_{10}\right)_{78} \mathrm{Si}_{12} \mathrm{~B}_{10}$ and $0.2 \mu \mathrm{m}$ AIN layers with Si substrate thickness adjusted for constant resonance frequency, i.e., $h_{s} \propto a^{2}$ for $h_{s} \gg h_{m}, h_{p}$. The five cases shown are $Q_{f}=100$ with $f=1 \mathrm{kHz}, Q_{f}=1000$ with $f=10$, 1 , and $0.1 \mathrm{kHz}$, and $Q_{f}=10000$ with $f=1 \mathrm{kHz}$, including the frequency-dependent substrate thickness behavior up to $50 \mu \mathrm{m}$. In all cases, detection limits decrease with greater ME cantilever size and approach a limit corresponding to the optimum layerthickness ratios of Fig. 4(h). Changes in resonance frequency do not affect the detection limit and produce cantilever-size changes scaling with $f^{-1 / 2}$. Quality factor changes mainly yield detection limit changes scaling as $Q_{f}^{-1 / 2}$ without affecting size.

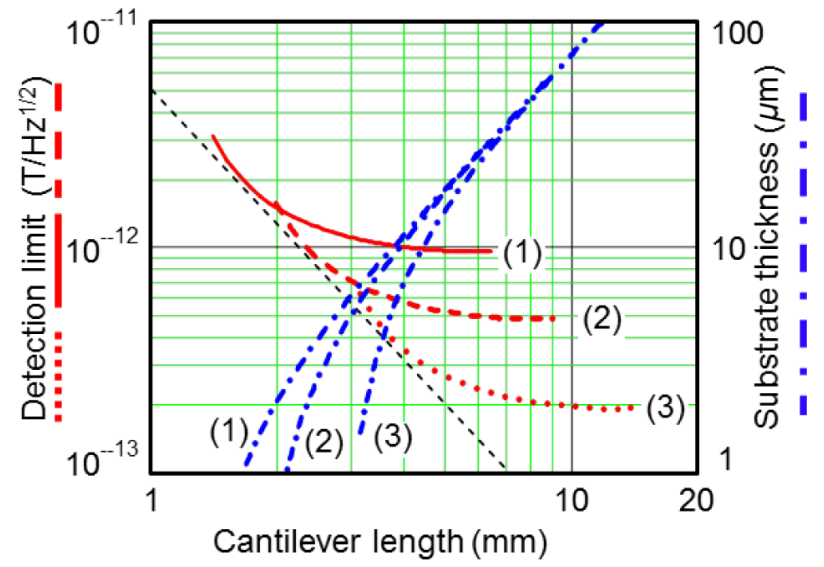

FIG. 7. Detection limit scaling with $\left(\mathrm{Fe}_{90} \mathrm{Co}_{10}\right)_{78} \mathrm{Si}_{12} \mathrm{~B}_{10} /$ $\mathrm{Si} / \mathrm{AlN}$ cantilever size (aspect ratio equals 3 ) for three cases of constant $\left(\mathrm{Fe}_{90} \mathrm{Co}_{10}\right)_{78} \mathrm{Si}_{12} \mathrm{~B}_{10}$ and AlN layer thicknesses, $Q_{f}=$ 1000 , and substrate thickness adjusted to yield $f=1 \mathrm{kHz}$. Cases are (1) $\left(\mathrm{Fe}_{90} \mathrm{Co}_{10}\right)_{78} \mathrm{Si}_{12} \mathrm{~B}_{10}=1.8 \mu \mathrm{m}$ and $\mathrm{AlN}=0.2 \mu \mathrm{m}$ with $\mathrm{Si}$ $\leq 30 \mu \mathrm{m}$, (2) $\left(\mathrm{Fe}_{90} \mathrm{Co}_{10}\right)_{78} \mathrm{Si}_{12} \mathrm{~B}_{10}=3.6 \mu \mathrm{m}$ and $\mathrm{AlN}=0.4 \mu \mathrm{m}$ with $\mathrm{Si} \leq 60 \mu \mathrm{m}$, and $(3)\left(\mathrm{Fe}_{90} \mathrm{Co}_{10}\right)_{78} \mathrm{Si}_{12} \mathrm{~B}_{10}=9 \mu \mathrm{m}$ and $\mathrm{AlN}=1.0 \mu \mathrm{m}$ with $\mathrm{Si} \leq 150 \mu \mathrm{m}$. Detection limit minima are found to scale as $1 / h_{m}$ and correspond to maximum ME cantilever lengths. Alternatively, minimum-size ME cantilevers can be achieved at the expense of greater detection limits. The black dashed line indicates the predicted detection-limit-minimumsize relation for the materials, 1st bending mode, $Q_{f}=1000$, and $f=1 \mathrm{kHz}$ investigated here, whereby the frequency and $Q_{f}$-scaling behavior of Fig. 6 applies (not shown).

to the piezoelectric constant. Thus, as expected, thermal vibrations occur independent of functional material properties, while the associated noise depends on $g_{31 p}$. For dominating thermal-vibration noise, the total noise density is essentially proportional to $g_{31}$. As a result, the detection limit (12) is independent of the piezoelectric constant and inversely proportional to the piezomagnetic constant for the thermal-vibration-noise-limited case and inversely proportional to the $g_{31 p} d_{33 m}$ product otherwise.

Applications for detection of oscillating magnetic fields require sensors for specific frequencies. Thus, ME sensors of the same resonance frequency should be compared when investigating the parameter space for low detection limits. This can be achieved by length scaling of cantilevers. We omit the results of length-only scaling here and proceed to proportional length and width scaling (constant aspect ratio of 3) of ME cantilevers with the results for $f=1 \mathrm{kHz}, Q_{f}=1000$, and the functional layer thicknesses of Fig. 2 shown in Fig. 3. Figure 3(a) shows the increasing length with substrate thickness for ME cantilevers scaled to $1 \mathrm{kHz}$ displaying a behavior proportional to $h_{s}^{1 / 2}$ for thick substrates. Figure 3(b) gives the corresponding behavior of ME coefficient and feedback capacity, both of which increase with sensor size for constant gain (equal to 10). The sensitivity is independent of 


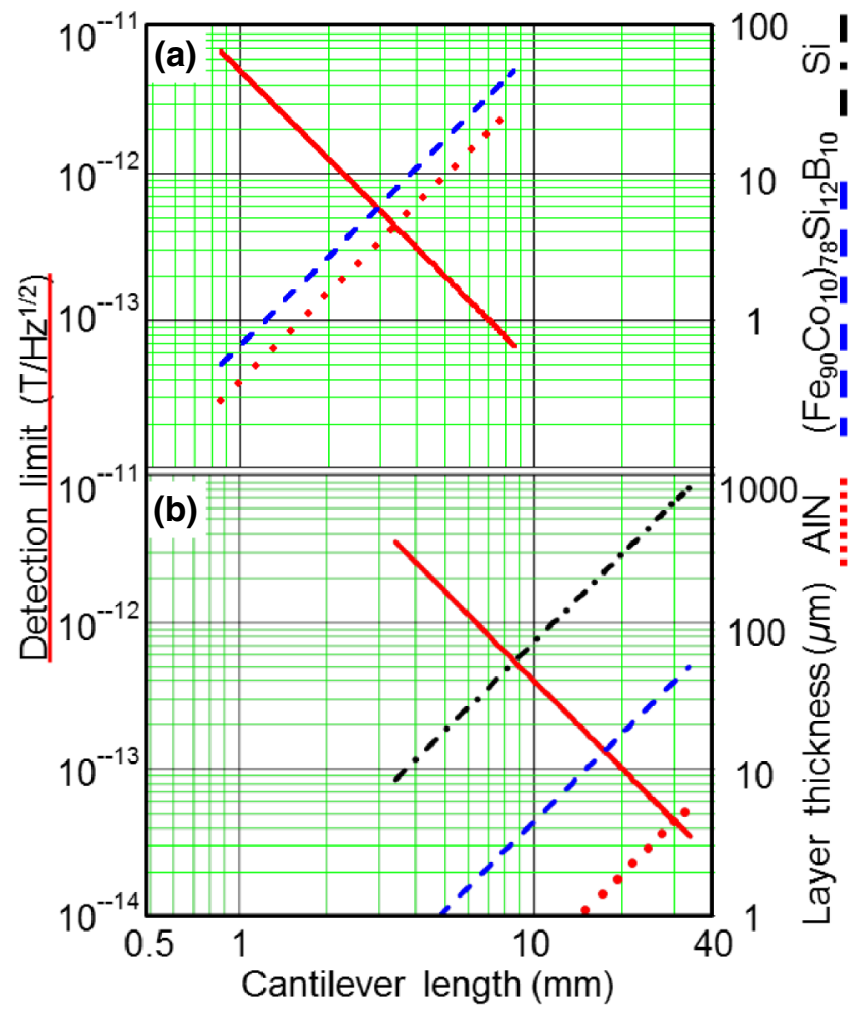

FIG. 8. Detection limit scaling with $\left(\mathrm{Fe}_{90} \mathrm{Co}_{10}\right)_{78} \mathrm{Si}_{12} \mathrm{~B}_{10} /$ $\mathrm{Si} / \mathrm{AlN}$ cantilever size using proportional layer thickness scaling and length and width scaling $a \propto h_{s}^{1 / 2}$ with constant aspect ratio of 3 to yield a constant $1 \mathrm{kHz}$ resonance frequency. The detection limit behavior for $Q_{f}=1000$ is given including the corresponding layer thicknesses for size-minimized ME cantilevers $(35 \%$ PE fraction, no substrate) [Fig. 8(a)], and for functional-layerthickness-minimized ME cantilevers without size constraint [Fig. 8(b)].

sensor size and thus identical to Fig. 2(b). As a surprise, also all corresponding noise components of scaled and unscaled cantilevers are identical for a given substrate thickness [Fig. 3(c)], showing that size and frequency effects of scaling compensate each other. As a result of unchanged sensitivity and noise behavior, the detection limits of scaled and unscaled cantilevers of corresponding substrate thickness are also identical [Fig. 3(d)]. Thus, at $h_{s}=25 \mu \mathrm{m}$, a $3 \times 1-\mathrm{mm}^{2}$ cantilever of $f=4 \mathrm{kHz}$ has the same detection limit of about $1 \mathrm{pT} / \mathrm{Hz}^{1 / 2}$ as a $6 \times 2-\mathrm{mm}^{2}$ cantilever at $f=1 \mathrm{kHz}$.

Next, we consider the effect of layer-thickness ratios on the detection limit of a magnetoelectric cantilever sensor with dominating thermal-vibration noise. This is important as process issues may narrow the available range of layer thicknesses. Furthermore, optimized ratios yield a minimum total layer thickness and thus cantilever size at a given resonance frequency. Figure 4 addresses the effects of varying thickness ratios of functional layer thicknesses on sensitivity, noise density, and detection limit. Displayed is the substrate thickness behavior of sensitivity, total noise density, and detection limit as a function of PE fraction [= $\left.h_{p} /\left(h_{p}+h_{m}\right)\right]$ and substrate thickness for the unscaled and size-scaled results corresponding to Figs. 2 and 3, respectively. Figure 4(a) displays the range of resonance frequencies for $3 \times 1-\mathrm{mm}^{2}$ cantilevers as a function of substrate thickness and Fig. 4(b) the corresponding size range for cantilevers scaled to $1 \mathrm{kHz}$. Figures 4(c) and 4(d) show the PE fraction and substrate-thickness behavior of the sensitivity being identical for unscaled and size-scaled cantilevers, as discussed in conjunction with Figs. 2 and 3. The sensitivity maximum near $35 \%$ PE fraction results from the short-circuit $\mathrm{ME}$ response and the inverse feedback capacity, which decrease and increase with PE fraction, respectively. Figures $4(\mathrm{e})$ and $4(\mathrm{f})$ display the thermalvibration-noise-dominated total noise behavior with $\mathrm{PE}$ fraction and substrate thickness, which is identical for the scaled and unscaled cases. The behavior results from the thermal noise amplitude of the cantilever being greatest at vanishing substrates, the strain-induced piezoelectric charge per amplitude $[(d Q / d H)(d H / d z)]$ with maximum near thick substrates, and the inverse sensor capacity increasing with PE fraction. The behavior of sensitivity and noise density differs in slope and gradient near thick substrates and low PE fractions. As a result, the detection limit minimum is produced near $10 \% \mathrm{PE}$ fraction and $25 \mu \mathrm{m}$ substrate thickness [Fig. 4(g)] and is identical for the size-scaled case [Fig. 4(h)]. This shows that PE fraction changes do not produce additional or lower detection limit minima. Comparing the unscaled and size-scaled cases, we find that the sensitivity, noise, and detection limit behavior with respect to $\mathrm{PE}$ fraction and substrate thickness dependence are identical. Thus, the layer-thickness ratios of Figs. 2 and 3 produce the lowest detection limits available for the resonance frequencies, quality factors, and materials investigated here. Figures $4(\mathrm{~g})$ and $4(\mathrm{~h})$ also give the PE fractions that minimize the detection limit for different substrate thicknesses, e.g., about 35\% for vanishing substrates.

Departing from the constraint of fixed total functional layer thicknesses, Fig. 5 displays the detection limit of unscaled $3 \times 1-\mathrm{mm}^{2} \mathrm{ME}$ cantilevers as a function of $\left(\mathrm{Fe}_{90} \mathrm{Co}_{10}\right)_{78} \mathrm{Si}_{12} \mathrm{~B}_{10}$ and $\mathrm{AlN}$ layer thickness for $50 \mu \mathrm{m} \mathrm{Si}$ substrates and $Q_{f}=1000$. The results show a strongly decreasing detection limit with increasing $\left(\mathrm{Fe}_{90} \mathrm{Co}_{10}\right)_{78} \mathrm{Si}_{12} \mathrm{~B}_{10}$ layer thickness if the AIN layer thickness exceeds a minimum of $10 \%$ of the $\left(\mathrm{Fe}_{90} \mathrm{Co}_{10}\right)_{78} \mathrm{Si}_{12} \mathrm{~B}_{10}$ thickness. The results show the importance of thick $\left(\mathrm{Fe}_{90} \mathrm{Co}_{10}\right)_{78} \mathrm{Si}_{12} \mathrm{~B}_{10}$ layers and maintaining at least $10 \% \mathrm{PE}$ fraction if low detection limits are to be achieved with minimum functional layer thickness. Exceeding the $10 \%$ PE fraction will only have a small effect toward lower detection limits.

In the following, we focus on the detection limit and cantilever-size effects which are important for 
localized magnetic field measurements and source field reconstruction with sensor arrays. Figure 6 displays detection limit results with size scaling (aspect ratio $a: b=$ 3 ) for constant resonance frequencies as a function of cantilever length $a$. Considering the behavior for $1 \mathrm{kHz}$ and $Q_{f}=1000$, we find the detection limit being proportional to $1 / a^{2}$ near vanishing substrate thickness and being almost independent of length at thick substrates. Five cases for constant $\left(\mathrm{Fe}_{90} \mathrm{Co}_{10}\right)_{78} \mathrm{Si}_{12} \mathrm{~B}_{10}$ and AlN layers are shown in conjunction with the corresponding substrate thickness behavior, i.e., $10 \mathrm{kHz}, 1 \mathrm{kHz}$, and $100 \mathrm{~Hz}$ for $Q_{f}=1000$ as well as $Q_{f}=100$, and $Q_{f}=10000$ for $1 \mathrm{kHz}$. The results show that size scaling to different resonance frequencies only affects cantilever size but not the detection limit behavior, whereby size scales as $f^{-1 / 2}$. Conversely, the results for different quality factors affect essentially only the detection limit but not the cantilever size. Here, the detection limit scales as $Q_{f}^{-1 / 2}$ as a result of the ME response and sensitivity being proportional to $Q_{f}^{1}$ [Eq. (5) and Wan et al. [9]] and the thermal-vibration noise to $Q_{f}^{1 / 2}$ [Eq. (9)]. As $Q_{f}$-independent amplifier and cable and $Q_{f}$-dependent thermal-vibration-noise densities approach similar values for thick substrates at $Q_{f}=1000$ [Fig. 3(c)], the slope of the total noise density changes. This produces an increasing detection limit for thick substrates, which is even more pronounced at $Q_{f}=100$ where amplifier and cable noise dominates. At $Q_{f}=$ 10000 , thermal-vibration noise greatly exceeds amplifier and cable noise and the increasing detection limit effect is absent. The above detection limit behavior is identical for $10 \mathrm{kHz}, 1 \mathrm{kHz}$, and $100 \mathrm{~Hz}$ cantilevers at $Q_{f}=1000$. It should be pointed out that amplifier and cable noise does not constitute a fundamental limit here and lower noise devices [45] are available to ensure thermal-vibrationnoise-limited behavior at low quality factors.

Figure 7 gives the detection limit and size effects for $Q_{f}=1000$ cantilevers size scaled to $1 \mathrm{kHz}$ if the constant functional layer and the maximum substrate thicknesses are increased 2-fold and 5-fold from the values of Fig. 6. The results show that increased functional layer thicknesses produce lower detection limits proportional to $1 / h_{m}$, while simultaneously increasing cantilever size as $h_{s}^{1 / 2}$. Proportional scaling of all layer thicknesses with associated cantilever size scaling for constant resonance frequency does not affect the ratio of noise components thus yielding thermal-vibration-noise-dominated detection limits for $Q_{f}=1000$. Furthermore, the numerically observed asymptotic $1 / a^{2}$ behavior of the detection limit near vanishing substrate thicknesses indicates the existence of a minimum size constraint of $\delta H \approx 5 \mathrm{pT} / \mathrm{Hz}^{1 / 2} / a^{2}$ for constant-aspect-ratio cantilevers with the length $a$ given in units of $\mathrm{mm}$.

Finally, Fig. 8 displays the detection limit versus cantilever size behavior for $1 \mathrm{kHz}$ and $Q_{f}=1000$ with proportional scaling of all layer thicknesses $h_{s}, h_{m}$, and $h_{p}$ and size scaling as $h_{s}^{1 / 2}$. Also shown are all layer thicknesses and detection limits for $\left(\mathrm{Fe}_{90} \mathrm{Co}_{10}\right)_{78} \mathrm{Si}_{12} \mathrm{~B}_{10}$ layers up to $50 \mu \mathrm{m}$. The thermal-vibration-noise-limited detection limit displays a $1 / a^{2}$ behavior with cantilever size. Figure 8 (a) gives the results corresponding to the minimum size constraint of Fig. 7. Further investigation yields that minimum-size cantilevers for a given detection limit and resonance frequency are produced with vanishing substrates and about $35 \%$ PE fraction as suggested by bending stiffness considerations and the results of Fig. 4(h). This yields a detection limit of $67 \mathrm{fT} / \mathrm{Hz}^{1 / 2}$ for $8.6 \times 2.9-$ $\mathrm{mm}^{2}$-size cantilevers with $50 \mu \mathrm{m}\left(\mathrm{Fe}_{90} \mathrm{Co}_{10}\right)_{78} \mathrm{Si}_{12} \mathrm{~B}_{10}$ and $28 \mu \mathrm{m}$ AlN. Figure 8(b) displays the case without size constraint where further detection limit reduction via increasing size is negligible. This case is achieved when using the layer-thickness ratios of the detection limit minimum of Fig. 4(h), i.e., 10\% PE fraction and a Si substrate to $\left(\mathrm{Fe}_{90} \mathrm{Co}_{10}\right)_{78} \mathrm{Si}_{12} \mathrm{~B}_{10}$ layer-thickness ratio of about 14. Here, $50 \mu \mathrm{m}\left(\mathrm{Fe}_{90} \mathrm{Co}_{10}\right)_{78} \mathrm{Si}_{12} \mathrm{~B}_{10}, 5.6 \mu \mathrm{m} \mathrm{AlN}$, and $830 \mu \mathrm{m}$ Si yield a predicted detection limit of $35 \mathrm{fT} / \mathrm{Hz}^{1 / 2}$, albeit at $30 \times 10 \mathrm{~mm}^{2}$ cantilever size. Changes in quality factor and resonance frequency for the scaling results of Fig. 8 yield the same shifts as displayed in Fig. 6 (not shown). The size-scaling results of Figs. 6 and 8 identify increasing layer thicknesses at specific ratios and the quality factor as the methods of choice for lowering the detection limit in the thermal-vibration-noise-limited regime. No absolute detection limit is predicted within the scope of our model. However, for large cantilever sizes, amplifier and cable or eventually sensor noise from dielectric fluctuations in the AIN layer will become dominant, thus changing the behavior of Fig. 8. Furthermore, the thermal-vibration noise dominated scaling behavior of Fig. 8 is based on layer-thickness-independent material parameters. Producing thick magnetostrictive layers may be limited by the buildup of magnetostatic energy, which leads to relaxation via magnetic domain formation and reduced magnetostriction.

\section{CONCLUSION}

We derived a theory of thermal-vibration noise in magnetoelectric-composite cantilevers and calculate the resonant magnetoelectric response and thermal noise behavior of $\left(\mathrm{Fe}_{90} \mathrm{Co}_{10}\right)_{78} \mathrm{Si}_{12} \mathrm{~B}_{10} / \mathrm{Si} / \mathrm{AlN}$ composites to determine the magnetic field detection limit for the first bending mode. Thermal vibrations are found to dominate the noise behavior, in comparison with Johnson-Nyquist noise from the AlN layer and electronic noise, and to determine the fundamental thermal detection limit for most cantilever dimensions. To identify magnetometry limits for ultrasensitive magnetoelectric composites, we present a systematic investigation of the scaling behavior of ME response, sensitivity, noise components, and 
detection limit with cantilever size, layer thicknesses, quality factor $Q_{f}$, and resonance frequency $f$, focusing on low frequency applications, although the results are general. We find detection limits scaling as $1 / h_{m}, 1 / h_{p}$, and $1 /$ size $^{2}$ with variable functional layer thicknesses for constant $f$ and $Q_{f}$, and as $Q_{f}^{-1 / 2}$ for constant size and $f$. Different layer-thickness ratios and detection limit behavior are identified for the size-minimized and functionallayer-thickness-minimized scaling cases. Exemplary predicted detection limits achievable with and without size constraint for $\left(\mathrm{Fe}_{90} \mathrm{Co}_{10}\right)_{78} \mathrm{Si}_{12} \mathrm{~B}_{10} / \mathrm{Si} / \mathrm{AlN}$ cantilevers are $67 \mathrm{fT} / \mathrm{Hz}^{1 / 2}$ for $8.6 \times 2.9 \mathrm{~mm}^{2}$-size and $35 \mathrm{fT} / \mathrm{Hz}^{1 / 2}$ for $30 \times 10 \mathrm{~mm}^{2}$-size, respectively, with $\left(\mathrm{Fe}_{90} \mathrm{Co}_{10}\right)_{78} \mathrm{Si}_{12} \mathrm{~B}_{10}$ layers of $50 \mu \mathrm{m}$ at $f=1 \mathrm{kHz}$ and $Q_{f}=1000$ based on experimental material parameters for thin (approximately 2-4 $\mu \mathrm{m}$ ) layers. No absolute fundamental detection limit is predicted within the scope of the model if the material parameters observed in experiments with thin layers and the quality factors can be realized at thick functional layers.

\section{ACKNOWLEDGMENTS}

This work is supported by the DFG Collaborative Research Center SFB1261.

\section{APPENDIX A: ME-RESPONSE MODEL}

This appendix reiterates the derivation of the resonant short-circuit magnetoelectric response Eq. (5) of a threelayer cantilever of length $a$, width $b$, and strain-coupled magnetostrictive, substrate, and piezoelectric layers of thickness $h_{m}, h_{s}$, and $h_{p}$ respectively [39]. However, all necessary terms are given explicitly including the ones needed for the thermal-vibration noise calculation. Thin additional layers, i.e., contacts, and seed and adhesion layers, are neglected. We start with the constitutive equations (1) -(4) from Sec. II.

The coordinate system used here (Fig. 1) describes the cantilever of length $a$ in the $x$ direction, width $b$ in the $y$ direction (not shown), and the fixed end at $x=0$, and the bending displacement $w(x)$ in the $z$ direction. The neutral plane position $d_{m s p}$, i.e., the $z$-coordinate of the multilayer cantilever, where the strain vanishes, equals the offset to the bottom of the layer stack. As a result of the $z$ coordinate originating at the neutral plane, the cantilever curvature $d^{2} w / d x^{2}$ produces a strain in $x$ direction that is directly proportional to $z$ without offset, i.e., $S_{x}=-z d^{2} w / d x^{2}$. The neutral plane position $d$ is calculated from the force balance in the $x$ direction, requiring the integrated stresses in the $x$ direction over all layers to vanish, i.e., $\int T_{x} d z=0$. The integrations are performed over the MS, substrate, and PE layers, whereby the stresses have been inserted from the constitutive equations (1)-(4). For the oscillating cantilever, the $H$ and $D$ field-induced stresses are small compared to the stresses produced by inertia at resonance and have been neglected for the calculation of the neutral plane. Here, $h$ denotes the layer thicknesses with subscripts $m, s$, and $p$ referring to magnetostrictive, substrate, and piezoelectric layers, respectively. This yields the distance $d_{m s p}$ from the bottom of the stack to the neutral plane position as

$$
\begin{aligned}
d_{m s p}= & {\left[s_{11 p} s_{11 s} h_{m}^{2}+2 s_{11 p} s_{11 s} h_{m} h_{p}+2 s_{11 p} s_{11 s} h_{m} h_{s}\right.} \\
& \left.+s_{33 m} s_{11 s} h_{p}^{2}+2 s_{11 p} s_{33 m} h_{p} h_{s}+s_{11 p} s_{33 m} h_{s}^{2}\right] \\
& {\left[2 h_{s} s_{11 p} s_{33 m}+2 h_{m} s_{11 p} s_{11 s}+2 h_{p} s_{33 m} s_{11 s}\right]^{-1} . }
\end{aligned}
$$

Integrating the electric field over the PE layer yields the strain-induced voltage, i.e., $\int E_{3} d z=V$. Again the constitutive equations for the PE layer and the strain-curvature linearity are used. This yields

$$
V(x)=\int_{-d_{m s p}}^{h_{p}-d_{m s p}}\left[z \frac{g_{31 p}}{s_{11 p}} \frac{d^{2} w}{d x^{2}}+\left(\frac{g_{31 p}^{2}}{s_{11 p}}+\beta_{33}\right) D_{3}\right] d z .
$$

Please note that $E=E(x, z), T=T(x, z)$, and $S=S(x, z)$ depend on $x$ and $z$, while $D_{3}=D_{3}(x), V=V(x)$, $d^{2} w / d x^{2}=\left(d^{2} w / d x^{2}\right)(x)$, and bending moment $M=$ $M(x)$ (introduced below) vary only with $x$. Equation (A2) can be solved for $D_{3}$, resulting in the following linear behavior with cantilever curvature $d^{2} w / d x^{2}$, potential $V$, and $H_{3}$. This results in

$$
D_{3}(x)=T_{1} \frac{d^{2} w}{d x^{2}}+T_{2} V+T_{3} H_{3} .
$$

Here, we introduce numbered terms $T_{j}$ (not to be confused with the stress $T$ ), which are functions of material properties and cantilever geometry. The terms are

$$
T_{1_{m s p}}=\frac{g_{31 p}\left(2 d_{m s p}-h_{p}\right)}{2 g_{31 p}^{2}+2 s_{11 p} \beta_{33}}
$$

and

$$
T_{2 m s p}=\frac{s_{11 p}}{h_{p}\left(g_{31 p}^{2}+s_{11 p} \beta_{33}\right)} .
$$

$T_{3}=0$ as the $D_{3}$ and $H_{3}$ field-induced shifts of the neutral plane are neglected here. Equation (A3) relates the electric displacement, i.e., the electric field originating from free charges only, to the cantilever curvature and the voltage across the piezoelectric layer. Further evaluation requires knowledge of the cantilever curvature $d^{2} w / d x^{2}$. This is obtained by considering the bending moment. 
The net bending moment $M$ of the layer stack results in a deflection of the cantilever along $z$. This is obtained by integrating the moment contributions from $x$-direction stresses over all layers, i.e., $M=\int z T_{x x} d z$, whereby the full constitutive equations (1)-(4) and the strain-curvature linearity are inserted. Subsequently, solving for the bending moment and factoring to terms $T_{i}$ originating from cantilever curvature, and $H$ and $\mathrm{D}$ fields yields

$$
M(x)=T_{4} \frac{d^{2} w}{d x^{2}}+T_{5} D_{3}+T_{6} H_{3} .
$$

Inserting $D_{3}$ from Eq. (A3) yields the bending moment in terms $T_{j}$ corresponding to cantilever curvature, voltage across the piezoelectric layer, and magnetic field, i.e.,

$$
M(x)=T_{7} \frac{d^{2} w}{d x^{2}}+T_{8} V+T_{6} H_{3}
$$

Here, $T_{7}, T_{8}$, and $T_{6}$ denote the bending stiffness of the multilayer composite, the bending moment from applied voltages, and magnetic fields, respectively. The terms $T_{6}$ and $T_{7}$ are

$$
T_{6_{m s p}}=-\frac{d_{33 m} h_{m}\left(h_{m}-2 d_{m s p}+2 h_{p}+2 h_{s}\right)}{2 s_{33 m}}
$$

and

$$
\begin{aligned}
T_{7_{m s p}}= & -\frac{h_{m}}{3 s_{33 m}}\left[3 d_{m s p}^{2}-3 d_{m s p} h_{m}-6 d_{m s p} h_{p}-6 d_{m s p} h_{s}\right. \\
& \left.+h_{m}^{2}+3 h_{m} h_{p}+3 h_{m} h_{s}+3 h_{p}^{2}+6 h_{p} h_{s}+3 h_{s}^{2}\right] \\
& -\frac{h_{s}}{3 s_{11 s}}\left[3 d_{m s p}^{2}-6 d_{m s p} h_{p}-3 d_{m s p} h_{s}+3 h_{p}^{2}\right. \\
& \left.+3 h_{p} h_{s}+3 h_{s}^{2}\right]+\frac{h_{p}}{3 s_{11 p}}\left[3 d_{m s p} h_{p}-3 d_{m s p}^{2}-h_{p}^{2}\right] \\
& +\frac{g_{31 p}^{2} h_{p}\left(h_{p}-2 d_{m s p}\right)^{2}}{4 g_{31 p}^{2} s_{11 p}+4 \beta_{33} s_{11 p}^{2}} .
\end{aligned}
$$

The term $T_{8_{m s p}}$ is identical to $T_{1_{m s p}}$. Using the bending stiffness term $T_{7}$ and a mass term of multilayer mass per unit area, the equation of motion for the bending displacement $W(x, t)$ of the cantilever in the $z$ direction in the Euler-Bernoulli beam theory approximation is

$$
0=T_{7} \frac{d^{4} W}{d x^{4}}+\left(h_{m} \rho_{m}+h_{s} \rho_{s}+h_{p} \rho_{p}\right) \frac{d^{2} W}{d t^{2}},
$$

where $\rho_{m}, \rho_{s}$, and $\rho_{p}$ denote the densities of the magnetostrictive, substrate, and piezoelectric layers, respectively. Please note that the Euler-Bernoulli theory neglects the $y$ dimension. However, the short-circuit ME response and noise depend on the cantilever width. The above equation of motion yields the known harmonic solution for the cantilever.

$$
W(x, t)=w(x) e^{i \omega t}
$$

with the general solution

$$
\begin{aligned}
w(x)= & C_{1} \cosh (k x)+C_{2} \sinh (k x) \\
& +C_{3} \cos (k x)+C_{4} \sin (k x) .
\end{aligned}
$$

Here, $k$ determines the bending modes and associated resonance frequencies as a result of the boundary conditions and corresponding characteristic equations. For the lowest bound-free bending mode, $k=1.875 / a$ with the resonance frequency of the three-layer cantilever of length $a$ being

$$
\omega=\frac{1.875^{2}}{a^{2}}\left(\frac{T_{7}}{h_{m} \rho_{m}+h_{s} \rho_{s}+h_{p} \rho_{p}}\right)^{1 / 2} .
$$

For the bound-free bending modes of the cantilever, the boundary conditions at the bound end $(x=0)$ are vanishing displacement and slope. At the free end $(x=a)$, both the bending moment and its gradient, i.e., the transverse shearing force, vanish. Solving the four equations of the boundary conditions yields $C_{1}=-C_{3}$ and $C_{2}=-C_{4}$ as well as

$$
C_{3}=\frac{\left(T_{6} H_{3}+T_{8} V\right)[\cosh (k a)+\cos (k a)]}{2 T_{7} k^{2}[\cosh (k a) \cos (k a)+1]} .
$$

and

$$
C_{4}=\frac{\left(T_{6} H_{3}+T_{8} V\right)[\sin (k a)-\sinh (k a)]}{2 T_{7} k^{2}[\cosh (k a) \cos (k a)+1]} .
$$

As a result, the specific solution for the bound-free cantilever is

$$
\begin{aligned}
w(x)= & \frac{\left(T_{6} H_{3}+T_{8} V\right)[\sin (k a)-\sinh (k a)]}{2 T_{7} k^{2}[\cosh (k a) \cos (k a)+1]} \\
& \{[\cosh (k a)+\cos (k a)][\cos (k x)-\cosh (k x)] \\
& +[\sinh (k a)-\sin (k a)][\sinh (k x)-\sin (k x)]\} .
\end{aligned}
$$

The motion-induced free piezoelectric charge is calculated by integrating the D field [Eq. (A3)] over the area of the layer. For full-area contacts, this yields $Q=$ $\int_{0}^{a} \int_{0}^{b} D_{3} d x d y$. Inserting Eq. (A3) and the above solution for $W(x)$ Eq. (A16) and solving the integral yields $Q$ as a linear equation in $V$ and $H_{3}$. For the short-circuit case 
TABLE I. Material parameters.

\begin{tabular}{lccc}
\hline \hline & $\left(\mathrm{Fe}_{90} \mathrm{Co}_{10}\right)_{78} \mathrm{Si}_{12} \mathrm{~B}_{10}{ }^{\mathrm{a}}$ & $\mathrm{Si}$ & $\mathrm{AlN}$ \\
\hline Density $\left(\mathrm{Kg} / \mathrm{m}^{3}\right)$ & 7250 & 2328 & 3268 \\
Elastic compliance & $s_{33 m}=$ & $s_{11 s}=$ & $s_{11 p}=$ \\
$\left(10^{-12} \mathrm{~Pa}^{-1}\right)$ & 7.738 & 5.974 & 2.899 \\
Piezoelectric constant & & & \\
$g_{31 p}\left(\mathrm{~m}^{2} / \mathrm{A}\right.$ s $)$ & & 0.024 \\
Piezomagnetic constant & & \\
$d_{33 m}(\mathrm{~m} / \mathrm{A})$ & $6.577 \times 10^{-8}$ & & \\
$\begin{array}{l}\text { Dielectric constant } \\
\beta_{33}(\mathrm{Vm} / \mathrm{A} \text { s })\end{array}$ & & & $1.27 \times 10^{10}$ \\
\hline \hline
\end{tabular}

${ }^{\mathrm{a}}\left(\mathrm{Fe}_{90} \mathrm{Co}_{10}\right)_{78} \mathrm{Si}_{12} \mathrm{~B}_{10}[12]$.

$V=0$ and the result of the motion-induced piezoelectric charge $Q$ yields

$$
\frac{d Q}{d H}=\frac{T_{1} T_{6} b[\cosh (k a) \sin (k a)+\sinh (k a) \cos (k a)]}{T_{7} k[1+\cosh (k a) \cos (k a)]},
$$

i.e., the short-circuit ME coefficient Eq. (6) without functional layer thickness and quality factor normalizations.

The $Q_{f}$ normalization in the solutions [Eq. (5)] is performed by taking $k$ corresponding to $1 / 10$ of the resonance frequencies where the $\mathrm{ME}$ responses are stable as Eq. (A17) diverges with $k$ being on resonance, i.e., $k=$ $1.875 / a$ or $4.694 / a \ldots$ for higher-order bending modes.

The material properties [12] used in the calculation are given in Table I.

\section{APPENDIX B: ME SENSOR NOISE MODEL}

This appendix gives further details of the noise density from thermal vibrations, dielectric fluctuations of the AIN layer, and amplifier electronics at the charge amplifier output [Eq. (8)] derived by Jahns [31] and reiterated for convenience below:

$$
\begin{aligned}
V_{\text {noise-SC }}= & {\left[\left(\frac{\left|Z_{f}\right|}{\left|Z_{\mathrm{ME}}\right|}\right)^{2}\left|\frac{1}{1+i 2 \pi f C_{\mathrm{ME}} R_{\mathrm{ME}}}\right|^{2} \frac{E_{\mathrm{ME}}{ }^{2}}{\Delta f}\right.} \\
& +\left(\frac{\left|Z_{f}\right|}{\left|Z_{\mathrm{ME}}\right|}\right)^{2} \frac{E_{\mathrm{TV}}{ }^{2}}{\Delta f}+\left|1+\frac{Z_{f}}{Z_{\mathrm{MEC}}}\right|^{2} E_{n}{ }^{2}+\left|Z_{f}\right|^{2} I_{n}{ }^{2} \\
& \left.+\left|\frac{1}{1+i 2 \pi f C_{f} R_{f}}\right|^{2} \frac{E_{f}{ }^{2}}{\Delta f}+\left|Z_{f}\right|^{2} \frac{E_{c}{ }^{2}}{R_{c}{ }^{2} \Delta f}\right]^{1 / 2} .
\end{aligned}
$$

Here, the first term denotes the noise density from the dielectric losses in the AlN layer. Because of their low resistance, corresponding Johnson-Nyquist noise from the $\left(\mathrm{Fe}_{90} \mathrm{Co}_{10}\right)_{78} \mathrm{Si}_{12} \mathrm{~B}_{10}$ and $\mathrm{Si}$ substrate layers are neglected. The second term gives the noise density from thermal cantilever vibrations [Eq. (10)] derived in Appendix C. The
TABLE II. Noise model parameters.

\begin{tabular}{lc}
\hline Temperature & $T=300 \mathrm{~K}$ \\
Loss tangent AIN [47] & $\tan \delta_{\mathrm{ME}}=0.00025$ \\
Loss tangent cable & $\tan \delta_{c}=0.0005$ \\
Capacitance cable & $C_{c}=15 \mathrm{pF}$ \\
Analog Devices AD745: & \\
Current noise at $1 \mathrm{kHz}^{\mathrm{a}}$ & $I_{n}=6.9 \times 10^{-15} \mathrm{~A} / \mathrm{Hz}^{1 / 2}$ \\
Voltage noise at $1 \mathrm{kHz}^{\mathrm{a}}$ & $E_{n}=3.2 \times 10^{-9} \mathrm{~V} / \mathrm{Hz}^{1 / 2}$ \\
\hline \hline
\end{tabular}

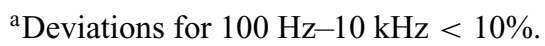

remaining four terms give the noise densities from the voltage and current noises of the amplifier (AD745), the feedback capacitance, and the coaxial cable connecting the ME composite and amplifier, respectively. Here, $Z_{\mathrm{ME}}, Z_{\mathrm{MEC}}$, and $Z_{f}$ denote the impedances of the ME sensor, ME sensor with cable, and feedback loop, respectively [31]. The impedances are $Z_{\mathrm{ME}}=\left(R_{\mathrm{ME}}^{-1}+2 \pi i f C_{\mathrm{ME}}\right)^{-1}, Z_{c}=\left(R_{c}^{-1}+\right.$ $\left.2 \pi i f C_{c}\right)^{-1}$, and $Z_{\mathrm{MEC}}=\left(Z_{\mathrm{ME}}^{-1}+Z_{c}^{-1}\right)^{-1}$ and for the feedback loop $Z_{f}=\left(R_{f}^{-1}+2 \pi i f C_{f}\right)^{-1}$ with $i=\sqrt{-1}, C_{f}=$ $C_{\mathrm{ME}} / G$, and gain $G=10$. Here, $C_{\mathrm{ME}}$ denotes the sensor capacity determined by the AIN layer thickness and cantilever size for full-area electrodes. This yields the dielectric losses of the AlN layer as $R_{\mathrm{ME}}=\left(2 \pi f C_{\mathrm{ME}} \tan \left(\delta_{\mathrm{ME}}\right)\right)^{-1}$ with $\tan \delta_{\mathrm{ME}}=0.00025$ for AlN. The associated noise voltage is $E_{\mathrm{ME}}=\left(4 k_{B} T \Delta f R_{\mathrm{ME}}\right)^{1 / 2}$ with Boltzmann constant $k_{B}$, temperature $T$, resonance frequency $f$, and noise bandwidth $\Delta f$. Cable noise voltage $E_{c}$ is calculated correspondingly from $C_{c}=15 \mathrm{pF}$ with $\tan \delta_{c}=0.0005$.

The thermal-vibration noise term is the only term with a strong noise bandwidth dependence in Eq. (B1) as [Eq. (9)] contains the quality factor. For the case of small noise bandwidths compared to the linewidth of the vibration mode considered in this work, the detection limit results are independent of the noise bandwidth. Noise model parameters are given in Table II.

\section{APPENDIX C: THERMAL-VIBRATION NOISE MODEL}

For the derivation of Eqs. (10) and (11), we use the result for $d Q / d H$ given in Eq. (A17) and calculate $d H / d z$. Evaluation of Eq. (A16) at the cantilever tip $x=a$ for the short-circuit case $V=0$ yields

$$
\left.w\right|_{x=a}=\frac{T_{6} H_{3}[\sin (k a) \sinh (k a)]}{T_{7} k^{2}[\cosh (k a) \cos (k a)+1]}
$$

and

$$
\left.\frac{d H}{d z}\right|_{x=a}=\frac{T_{7} k^{2}[\cosh (k a) \cos (k a)+1]}{T_{6}[\sin (k a) \sinh (k a)]}
$$

which in conjunction with Eq. (A17) results in Eq. (10).

For the ME cantilever, $k_{j}=211.6 T_{7} b a^{-3}$ is the modedependent force constant, $m_{j}=17.12 a b\left(h_{p} \rho_{p}+h_{m} \rho_{m}\right.$ 
$\left.+h_{s} \rho_{s}\right)$ the modal mass, and $\omega_{j}=\left(k_{j} / m_{j}\right)^{0.5}$ the resonance frequency. Here, $\phi_{j}=-8.27$ and $c_{j}=\left(k_{j} m_{j}\right)^{0.5}$ $Q_{f}^{-1}$ for the first bound-free bending mode $j=1$ with $h_{s}$ being the substrate thickness and $\rho_{p}, \rho_{m}$, and $\rho_{s}$ the layer specific densities.

[1] M. Fiebig, Revival of the magnetoelectric effect, J. Phys. D: Appl. Phys. 38, R123 (2005).

[2] W. Eerenstein, N. D. Mathur, and J. F. Scott, Multiferroic and magnetoelectric materials, Nat. Rev. 442, 759 (2006).

[3] C. W. Nan, M. I. Bichurin, Shuxiang Dong, D. Viehland, and G. Srinivasan, Multiferroic magnetoelectric composites: Historical perspective, status, and future directions, J. Appl. Phys. 103, 031101 (2008).

[4] J. P. Velev, S. S. Jaswal, and E. Y. Tsymbal, Multi-ferroic and magnetoelectric materials and interfaces, Phil. Trans. R. Soc. A 369, 3069 (2011).

[5] J. Ma, J. Hu, Z. Li, and C. W. Nan, Recent progress in multiferroic magnetoelectric composites: From bulk to thin films, Adv. Mater. 23, 1062 (2011).

[6] A. A. Bukharaev, A. K. Zvezdin, A. P. Pyatakov, and Y. K. Fetisov, Straintronics: A new trend in micro- and nanoelectronics and materials science, Phys. Uspekhi 61, 1175 (2018).

[7] N. A. Spaldin and R. Ramesh, Advances in magnetoelectric multiferroics, Nat. Mater. 18, 203 (2019).

[8] D. L. Fox, D. R. Tilley, J. F. Scott, and H. J. Guggenheim, Magnetoelectric phenomena in $\mathrm{BaMnF}_{4}$ and $\mathrm{BaMn}_{0.99} \mathrm{Co}_{0.01} \mathrm{~F}_{4}$, Phys. Rev. B 21, 2926 (1980).

[9] J. G. Wan, Z. Y. Li, Y. Wang, M. Zeng, G. H. Wang, and J. M. Liu, Strong flexural resonant magnetoelectric effect in Terfenol-D /epoxy- $\mathrm{Pb}(\mathrm{Zr}, \mathrm{Ti}) \mathrm{O}_{3}$ bilayer, Appl. Phys. Lett. 86, 202504 (2005).

[10] N. Tiercelin, V. Preobrazhensky, P. Pernod, and A. Ostaschenko, Enhanced magnetoelectric effect in nanostructured magnetostrictive thin film resonant actuator with field induced spin reorientation transition, Appl. Phys. Lett. 92, 062904 (2008).

[11] P. Zhao, Z. Zhao, D. Hunter, R. Suchoski, C. Gao, S. Mathews, M. Wuttig, and I. Takeuchi, Fabrication and characterization of all-thin-film magnetoelectric sensors, Appl. Phys. Lett. 94, 243507 (2009).

[12] H. Greve, E. Woltermann, H. J. Quenzer, B. Wagner, and E. Quandt, Giant magnetoelectric coefficients in $\left(\mathrm{Fe}_{90} \mathrm{Co}_{10}\right)_{78} \mathrm{Si}_{12} \mathrm{~B}_{10}$-AIN thin film composites, Appl. Phys. Lett. 96, 182501 (2010).

[13] S. Marauska, V. Hrkac, T. Dankwort, R. Jahns, H. J. Quenzer, R. Knöchel, L. Kienle, and B. Wagner, Sputtered thin film piezoelectric aluminum nitride as a functional MEMS material, Microsyst. Technol. 18, 787 (2012).

[14] C. Kirchhof, M. C. Krantz, I. Teliban, R. Jahns, S. Marauska, B. Wagner, R. Knöchel, M. Gerken, D. Meyners, and E. Quandt, Giant magnetoelectric effect in vacuum, Appl. Phys. Lett. 102, 232905 (2013).

[15] J. Petrie, D. Viehland, D. Gray, S. Mandal, G. Sreenivasulu, G. Srinivasan, and A. S. Edelstein, Enhancing the sensitivity of magnetoelectric sensors by increasing the operating frequency, J. Appl. Phys. 110, 124506 (2011).
[16] R. Jahns, H. Greve, E. Woltermann, E. Quandt, and R. Knöchel, Sensitivity enhancement of magnetoelectric sensors through frequency-conversion, Sensor Actuat. A 183, 16 (2012).

[17] E. Yarar, S. Salzer, V. HrKac, A. Piorra, M. Höft, R. Knöchel, L. Kienle, and E. Quandt, Inverse bilayer magnetoelectric thin film sensor, Appl. Phys. Lett. 109, 022901 (2016).

[18] V. Röbisch, S. Salzer, N. O. Urs, J. Reermann, E. Yarar, C. Kirchhof, E. Lage, M. Hoeft, G. U. Schmidt, R. Knöchel, J. McCord, E. Quandt, and D. Meyners, Pushing the detection limit of thin film magnetoelectric heterostructures, J. Mat. Res. 32, 1009 (2017).

[19] K. Sekihara and B. Scholz, Generalized Wiener estimation of three-dimensional current distribution from biomagnetic measurements, IEEE Trans. Biomed. Eng. 43, 281 (1996).

[20] C. Cai, K. Sekihara, and S. S. Nagarajan, Hierarchical multiscale Bayesian algorithm for robust MEG/EEG source reconstruction, NeuroImage 183, 698 (2018).

[21] A. M. Lozano, N. Lipsman, H. Bergman, P. Brown, S. Chabardes, J. W. Chang, K. Matthews, C. C. McIntyre, T. E. Schlaepfer, M. Schulder, Y. Temel, J. Volkmann, and J. K. Krauss, Deep brain stimulation: Current challenges and future directions, Nat. Rev. Neurol. 15, 148 (2019).

[22] More than 150000 patients treated with DBS worldwide (L. R. Sankary, P. J. Ford, A. G. Machado, L. J. Hoeksema, R. V. Samala, and D. J. Harris: Deep brain stimulation at end of life: Clinical and ethical considerations. J. Palliat. Med., 23: 582 (2019).

[23] T. D. Stowe, K. Yasumura, T. W. Kenny, D. Botkin, K Wago, and D. Rugar, Attonewton force detection using ultrathin silicon cantilevers, Appl. Phys. Lett. 71, 228 (1997).

[24] Z. Li, K. Jiang, F. Khan, A. Goswami, J. Liu, A. Passian, and T. Thundat, Anomalous interfacial stress generation during sodium intercalation/extraction in $\mathrm{MoS}_{2}$ thin-film anodes, Sci. Adv. 5, eaav2820 (2019).

[25] E. Finot, A. Fabre, A. Passian, and T. Thundat, Dynamic and Static Manifestation of Molecular Absorption in Thin Films Probed by a Microcantilever, Phys. Rev. Appl. 1, 024001 (2014).

[26] H. B. Callen and T. A. Welton, Irreversibility and generalized noise, Phys. Rev. 83, 34 (1951).

[27] Z. Xing, J. Li, and D. Viehland, Modeling and the signalto-noise ratio research of magnetoelectric sensors at low frequency, Appl. Phys. Lett. 91, 142905 (2007).

[28] Z. Xing, J. Li, and D. Viehland, Noise and scale effects on the signal-to-noise ratio in magnetoelectric laminate sensor/detection units, Appl. Phys. Lett. 91, 182902 (2007).

[29] X. Zhuang, C. Cordier, S. Saez, M. Lam Chok Sing, C. Dolabdjian, J. Gao, J. F. Li, and D. Viehland, Theoretical analysis of the intrinsic magnetic noise spectral density of magnetostrictive-piezoelectric laminated composites, J. Appl. Phys. 109, 124512 (2011).

[30] R. Jahns, H. Greve, E. Woltermann, E. Quandt, and R. Knöchel, Noise performance of magnetometers with resonant thin-film magnetoelectric sensors, IEEE Trans. Inst. Meas. 60, 2995 (2011).

[31] R. Jahns, Untersuchung und Optimierung von Empfindlichkeit und Rauschverhalten magnetoelektrischer Sensoren. Ph.D. Thesis, Kiel University, p. 159, 2013. 
[32] X. Zhuang, M. Lam Chok Sing, C. Dolabdjian, Y. Wang, P. Finkel, J. Li, and D. Viehland, Mechanical noise limit of a strain-coupled Magneto(Elasto)electric sensor operating under a magnetic or an electric field modulation, IEEE. Sens. J. 15, 1575 (2015).

[33] J. L. Gugat, M. C. Krantz, J. Schmalz, and M. Gerken, Signal-to-noise ratio in cantilever magnetoelectric sensors, IEEE Trans. Magn. 52, 7005005 (2016).

[34] M. I. Bichurin, D. A. Filippov, V. M. Petrov, V. M. Laletsin, N. Paddubnaya, and G. Srinivasan, Resonance magnetoelectric effects in layered magnetostrictive-piezoelectric composites, Phys. Rev. B. 68, 132408 (2003).

[35] M. C. Krantz and M. Gerken, Theory of magnetoelectric effect in multilayer nanocomposites on a substrate: Static bending-mode response, AIP. Adv. 3, 022103 (2013).

[36] M. C. Krantz and M. Gerken, Theory of magnetoelectric effect in multilayer nanocomposites on a substrate: Resonant bending-mode response, AIP. Adv. 3, 052131 (2013).

[37] M. C. Krantz, J. L. Gugat, and M. Gerken, Resonant magnetoelectric response of cantilevers with magnetostrictive and piezoelectric layers on opposite sides of the substrate, AIP. Adv. 3, 062135 (2013).

[38] M. C. Krantz and M. Gerken, Static magnetoelectric and magnetoelastic response of composite cantilevers: Theory of short vs. open circuit operation and layer sequence effects, AIP. Adv. 5, 117229 (2015).

[39] M. C. Krantz, J. L. Gugat, and M. Gerken, Resonant magnetoelectric response of composite cantilevers: Theory of short vs. open circuit operation and layer sequence effects, AIP. Adv. 5, 117230 (2015).

[40] M. Gerken, Resonance line shape, strain and electric potential distributions of composite magnetoelectric sensors, AIP. Adv. 3, 062115 (2013).

[41] T. B. Gabrielson, Mechanical-thermal noise in micromachined acoustic and vibration sensors, IEEE Trans. Electr. Dev. 40, 903 (1993).
[42] H. J. Butt and M. Jaschke, Calculation of thermal noise in atomic force microscopy, Nanotechnology 6, 1 (1995).

[43] M. V. Salapaka, H. S. Bergh, J. Lai, A. Majumdar, and E. McFarland, Multi-mode noise analysis of cantilevers for scanning probe microscopy, J. Appl. Phys. 81, 2480 (1997).

[44] M. C. Krantz and M. Gerken, Effect of excitation mode on the magnetic field detection limit of magnetoelectriccomposite cantilevers, AIP. Adv. 10, 045108 (2020).

[45] P. Durdaut, S. Salzer, J. Reermann, V. Roebisch, P. Hayes, A. Piorra, D. Meyners, E. Quandt, G. Schmidt, R. Knöchel, and M. Hoeft, Thermal-mechanical noise in resonant thinfilm magnetoelectric sensors, IEEE. Sens. J. 17, 2338 (2017).

[46] F. Martin and P. Muralt, Thickness dependence of the properties of highly c-axis textured AlN thin films, J. Vac. Sci. Technol. A22, 361 (2004).

[47] E. Yarar, V. HrKac, C. Zamponi, A. Piorra, L. Kienle, and E. Quandt, Low temperature aluminum nitride thin films for sensory applications, AIP. Adv. 6, 075115 (2016).

[48] P. Durdaut, V. Penner, C. Kirchhof, E. Quandt, R. Knöchel, and M. Hoeft, Noise of a JFET charge amplifier for piezoelectric sensors, IEEE. Sens. J. 17, 7364 (2017).

[49] J. E. Sader, Frequency response of cantilever beams immersed in viscous fluids with applications to the atomic force microscope, J. Appl. Phys. 84, 64 (1998).

[50] C. Zener, Internal friction in solids II. General theory of thermoelastic internal friction, Phys. Rev. 53, 90 (1938).

[51] Z. Hao, A. Erbil, and F. Ayazi, An analytical model for support loss in micromachined beam resonators with inplane flexural vibrations, IEEE Sensors Actuat. 109, 156 (2003).

[52] G. Herzer, Magnetomechanical damping in amorphous ribbons with uniaxial anisotropy, Mat. Sci. Eng. A 226, 631 (1997).

[53] T. L. Gilbert, A phenomenological theory of damping in ferromagnetic materials, IEEE Trans. Magn. 40, 3443 (2004) 